\title{
Computers
}

\& Structures

PERGAMON

\section{An evaluation of the MITC shell elements}

\author{
Klaus-Jürgen Bathe ${ }^{\mathrm{a}, *}$, Alexander Iosilevich ${ }^{\mathrm{a}}$, Dominique Chapelle ${ }^{\mathrm{b}}$ \\ ${ }^{a}$ Massachusetts Institute of Technology, Department of Mechanical Engineering, Cambridge, MA 02139, USA \\ bINRIA-Rocquencourt, BP 105, 78153 Le Chesnay Cedex, France
}

Accepted 10 August 1999

\begin{abstract}
Based on fundamental considerations for the finite element analysis of shells, we evaluate in the present paper the performance of the MITC general shell elements. We give the results obtained in the analysis of judiciously selected test problems and conclude that the elements are effective for general engineering applications. (C) 2000 Elsevier Science Ltd. All rights reserved.
\end{abstract}

Keywords: Shell elements; Mixed interpolation; MITC elements; Benchmark problems

\section{Introduction}

Shell structures are encountered in many engineering designs, and the accurate stress analysis of these structures is frequently required. A major difficulty in such analyses is that different shell structures can behave very differently depending on the shell geometry and boundary conditions used [1]. Finite elements for the analysis of shells must therefore be able to effectively capture different shell behaviors with varied and complex stress conditions. Because of these challenges in shell analyses, numerous finite elements have been proposed as improved analysis procedures. However, only very few elements can be recommended for general use in engineering practice.

In an attempt to provide a basis for a deeper study of the currently available shell finite elements, and the development of improved discretization procedures, we presented some fundamental considerations for the finite element analysis of shell structures in Ref. [1].

* Corresponding author. Tel.: +1-617-253-6645; fax: +1617-253-2275.
We summarized the different characteristic behaviors of shell structures, discussed the difficulties encountered in finite element analysis of such structures, and finally presented an evaluation strategy of finite element procedures. Our objective in this paper is to apply this evaluation strategy to the MITC shell elements [2-4], and discuss the results obtained.

In the following sections of the paper, we first briefly review the difficulties encountered in the analysis of shell structures and in the development of general shell finite elements. We then evaluate the MITC shell elements in the light of the general shell analysis requirements by solving the test problems proposed in Ref. [1]. The results of these analyses show that the MITC elements provide effective discretization schemes for the general analysis of shell structures.

\section{The basic shell analysis problem}

When studying the shell analysis problem, it is expedient to consider first the underlying mathematical model and then the finite element solution of that model $[1,2]$. 


\subsection{On the mathematical model}

The general finite element analysis approach for shell structures is to use shell elements that are formulated based on general three-dimensional continuum theory and kinematic and stress assumptions [2]. The shell assumptions are those of Naghdi shell theory, that the normal stress through the thickness of the shell vanishes and that straight fibers originally normal to the midsurface remain straight during the deformations of the shell [5]. The "underlying shell mathematical model" of the general continuum-mechanics based finite element discretizations is derived in Ref. [6], where the differences to the Naghdi shell model are also enumerated.

In practice, the difficulties in shell analysis are most pronounced when the shell is thin. For this reason, in theoretical discussions, the case of the shell thickness $t$ being small is considered (including the limit case $t \rightarrow 0$ ), and in the numerical evaluation of shell elements, thin shell analysis is considered. Since the underlying shell mathematical model of the general shell finite element analysis approach is equivalent to the Naghdi shell theory when $t$ is small (and indeed the same limit problems are obtained when $t \rightarrow 0$, see Ref. [6]), we can use the Naghdi shell theory to identify the analysis difficulties and develop an appropriate evaluation strategy for shell elements.

Using the Naghdi shell theory, the general shell analysis problem is:

Find $\boldsymbol{U}_{t} \in \mathscr{U}$ such that

$$
t^{3} A\left(\boldsymbol{U}_{t}, \boldsymbol{V}\right)+t D\left(\boldsymbol{U}_{t}, \boldsymbol{V}\right)=G(\boldsymbol{V}) \quad \forall \boldsymbol{V} \in \mathscr{U}
$$

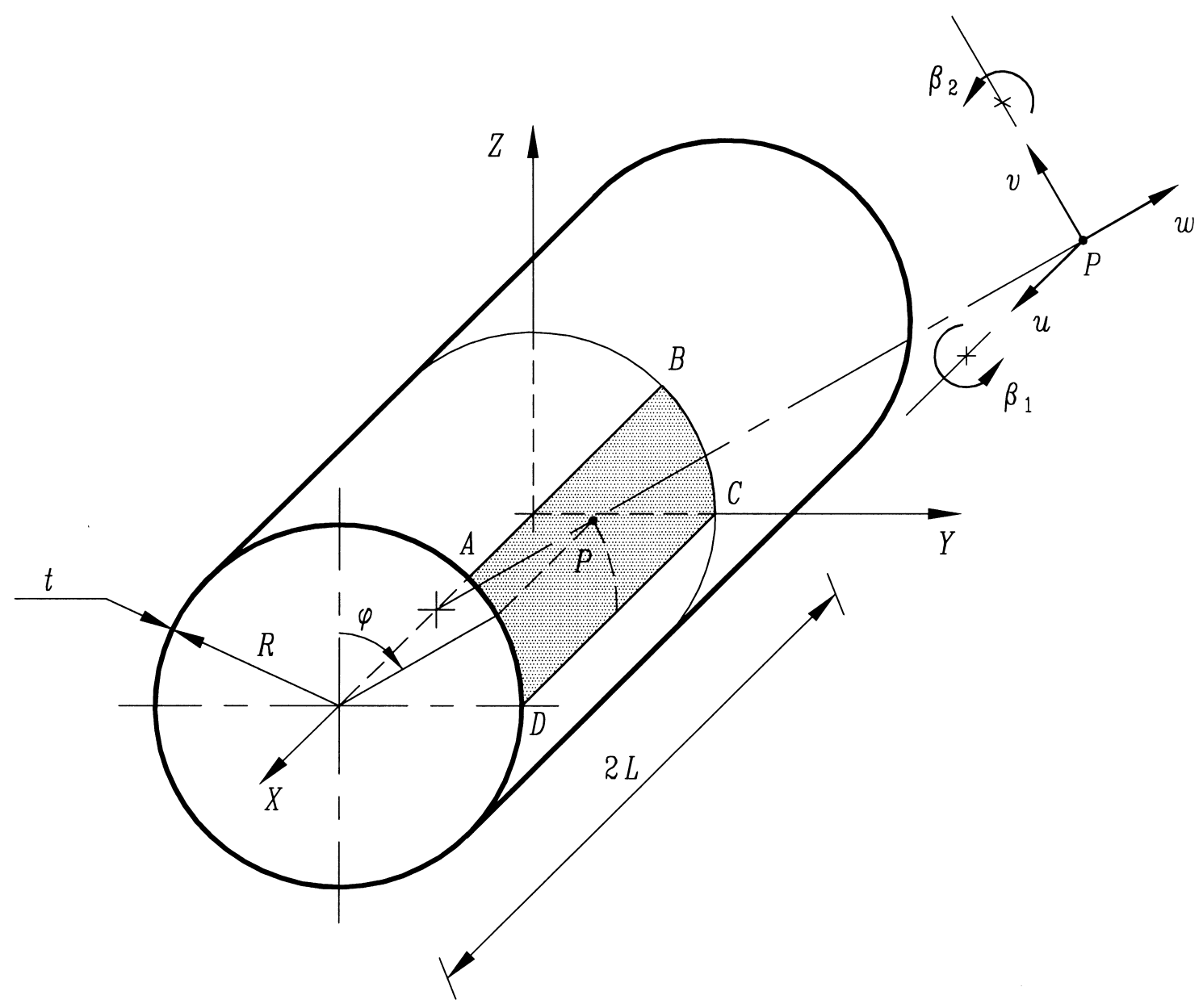

Fig. 1. Cylindrical shell. 
where

$\mathscr{U}=\left\{\boldsymbol{V}=(\boldsymbol{v}, \boldsymbol{\eta}), \boldsymbol{v} \in\left[H^{1}(\Omega)\right]^{3}, \boldsymbol{\eta} \in\left[H^{1}(\Omega)\right]^{2}\right\} \cap \mathscr{B} \mathscr{C}$

and $\boldsymbol{v}$ is the displacement vector of the shell midsurface, $\boldsymbol{\eta}$ lists rotations of the sections (originally normal to the midsurface), and $\mathscr{B} \mathscr{C}$ symbolically denotes the essential boundary conditions. The bilinear forms $A(\cdot, \cdot)$ and $D(\cdot, \cdot)$ capture, respectively, the bending and membrane/shear strain energies.

As we have discussed in Ref. [1], in the asymptotic analysis (i.e., when $t \rightarrow 0$ ), the following subspace, $\mathscr{U}_{0}$ takes on a crucial role:

$\mathscr{U}_{0} \stackrel{\text { def }}{=}\{\boldsymbol{V} \in \mathscr{U} \mid D(\boldsymbol{V}, \boldsymbol{V})=0\}$.

This subspace contains all those displacement patterns for which the membrane and shear strains are zero, hence, it is the subspace of pure bending displacements (also referred to as the subspace of inextensional displacements).

An essential specificity of shells is that $\mathscr{U}_{0}$ may be trivial, i.e., $\mathscr{U}_{0}=\{\boldsymbol{0}\}$. Such a situation is designated to be the case of "inhibited pure bending". The asymptotic behavior of a shell structure is highly dependent on whether or not pure bending is inhibited. Hence, to evaluate shell finite elements, test problems for which pure bending is inhibited $\left(\mathscr{U}_{0}=\mathbf{0}\right)$ and is not inhibited $\left(\mathscr{U}_{0} \neq\{\mathbf{0}\}\right)$ should be considered. In each case, the behavior of the finite element discretization should be measured as the thickness of the shell is decreased.

Considering the case of non-inhibited pure bending, we recognize that for the solution of Eq. (1) to remain both bounded and non-vanishing, we must assume the right-hand side to be of the form

$G(\boldsymbol{V})=t^{3} F_{b}(\boldsymbol{V})$,

so that for each value of $t$ the problem to be solved is:

Find $\boldsymbol{U}_{t} \in \mathscr{U}$ such that

$A\left(\boldsymbol{U}_{t}, \boldsymbol{V}\right)+\frac{1}{t^{2}} D\left(\boldsymbol{U}_{t}, \boldsymbol{V}\right)=F_{b}(\boldsymbol{V}) \quad \forall \boldsymbol{V} \in \mathscr{U}$.

When $t$ is very small, the membrane/shear term appears in this problem as a penalty term and for $t \rightarrow 0$ the solution of the following problem is approached:

Find $\boldsymbol{U}_{0}^{b} \in \mathscr{U}_{0}$ such that

$A\left(\boldsymbol{U}_{0}^{b}, \boldsymbol{V}\right)=F_{b}(\boldsymbol{V}) \quad \forall \boldsymbol{V} \in \mathscr{U}_{0}$

Furthermore, we have [1]

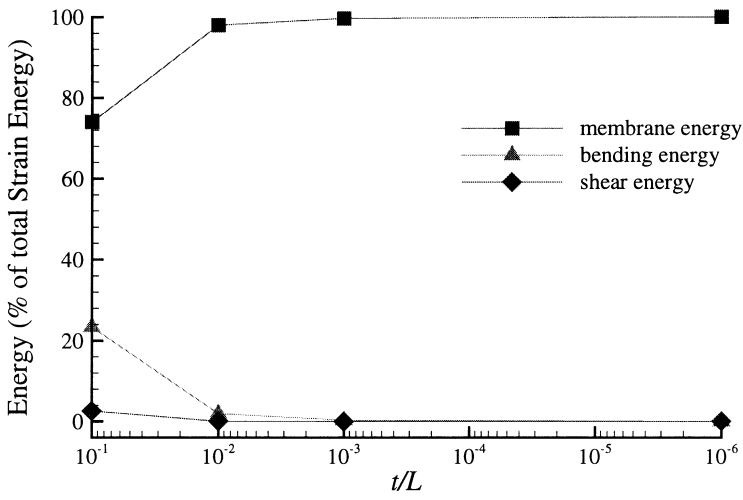

Fig. 2. Distribution of strain energy as a function of $t / L$ for the clamped cylindrical shell

$\lim _{t \rightarrow 0} \frac{1}{t^{2}} D\left(\boldsymbol{U}_{t}, \boldsymbol{U}_{t}\right)=0$.

The above considerations mean in practice, and in the evaluation of shell elements, that considering a pure bending non-inhibited problem, as the thickness approaches zero, the problem remains well-posed and the shear and membrane strains become negligible. Shell structures that carry loads primarily in bending are also referred to as bending-dominated shells.

The situation is quite different when a pure bending inhibited shell structure is analyzed. In this case the proper scaling of the loading is

$G(\boldsymbol{V})=t F_{m}(\boldsymbol{V})$

because the stiffness of the shell is proportional to the thickness as $t$ becomes small. The problem sequence to solve is:

Find $\mathbf{U}_{\mathrm{t}} \in \mathscr{U}$ such that

$t^{2} A\left(\boldsymbol{U}_{t}, \boldsymbol{V}\right)+D\left(\boldsymbol{U}_{t}, \boldsymbol{V}\right)=F_{m}(\boldsymbol{V}) \quad \forall \boldsymbol{V} \in \mathscr{U}$,

and the corresponding limit problem is:

Find $\boldsymbol{U}_{0}^{m} \in \mathscr{W}$ such that

$D\left(\boldsymbol{U}_{0}^{m}, \boldsymbol{V}\right)=F_{m}(\boldsymbol{V}) \quad \forall \boldsymbol{V} \in \mathscr{W}$,

where $\mathscr{W}$ is a space larger than $\mathscr{U}$ because strictly we no longer need continuity in the transverse displacements and section rotations. However, we require that

$\left|F_{m}(\boldsymbol{V})\right|^{2} \leq c D(\boldsymbol{V}, \boldsymbol{V}) \quad \forall \boldsymbol{V} \in \mathscr{W}$,

where $c$ is a constant. This relation ensures that the applied loading can be resisted by membrane stresses 


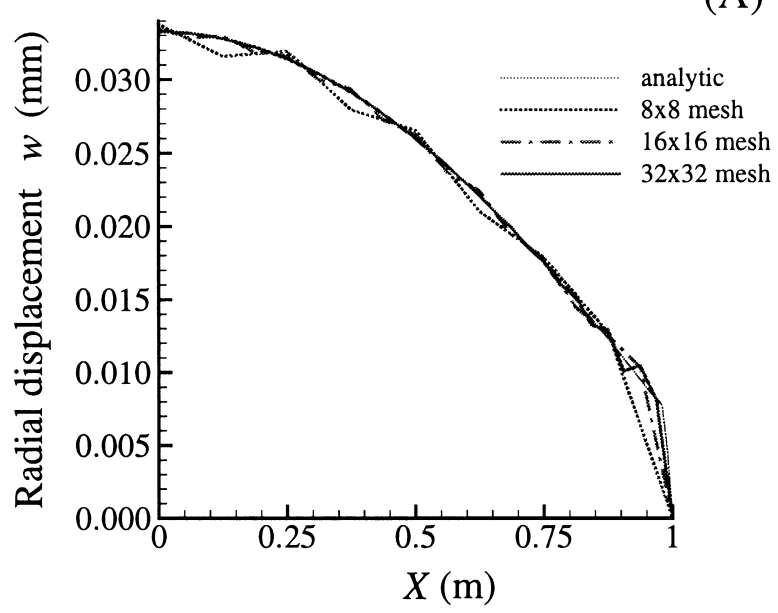

(B)


(D)
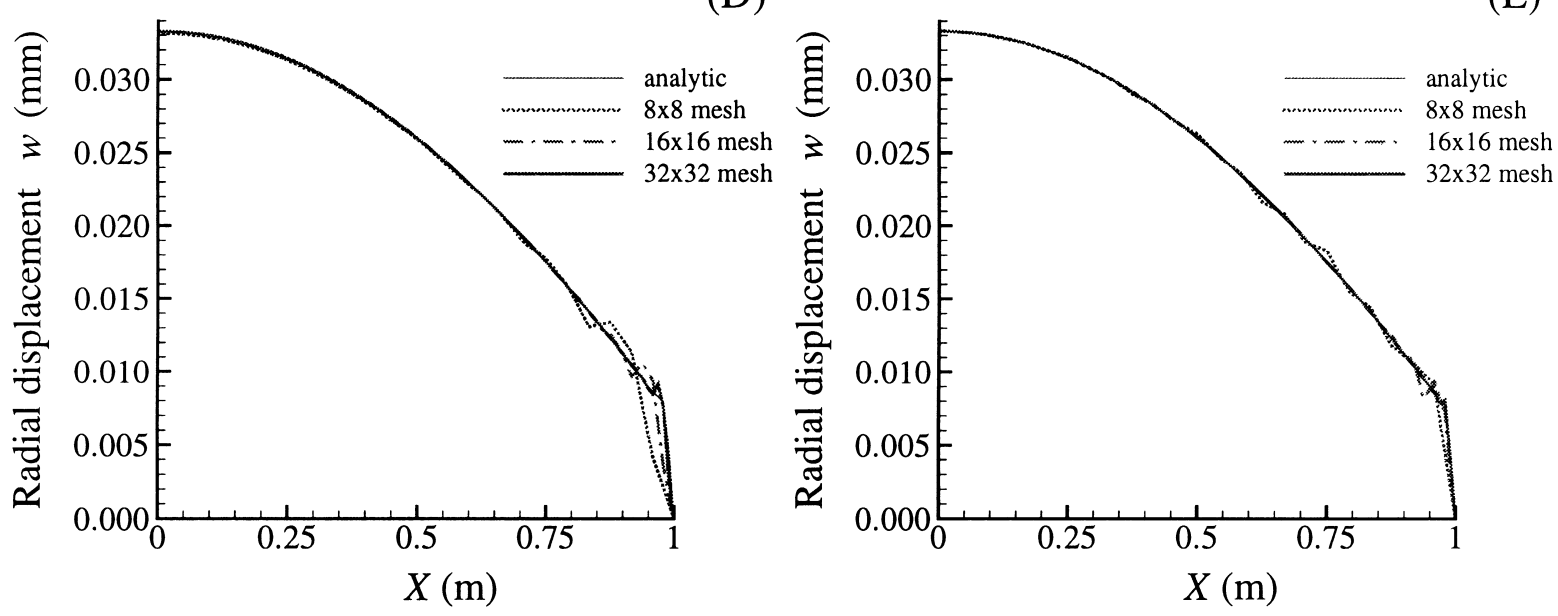

Fig. 3. Axial profile of radial displacement $w$ calculated along line $\mathrm{CD}$ for the clamped cylinder problem using $L=R=1 \mathrm{~m}$; $t / L=1 / 10,000 ; E=2 \times 10^{5} \mathrm{MPa} ; v=1 / 3 ; p_{0}=1 \mathrm{MPa}$. (A) MITC4 element; (B) QUAD9; (C) MITC9; (D) QUAD16; (E) MITC16. 
only. Shell structures that carry loads primarily in membrane action are also referred to as membranedominated shells.

\subsection{The finite element formulation}

For the finite element solution, a natural way to proceed is to use displacement/rotation interpolations. Using conforming interpolations, we select $\mathscr{U}^{h} \subset \mathscr{U}$, where $\mathscr{U}^{h}$ signifies the finite element space ( $h$ denoting the characteristic element size), and the finite element problem corresponding to Eq. (1) is:

Find $\boldsymbol{U}^{h} \in \mathscr{U}^{h}$ such that

$$
t^{3} A\left(\boldsymbol{U}^{h}, \boldsymbol{V}\right)+t D\left(\boldsymbol{U}^{h}, \boldsymbol{V}\right)=G(\boldsymbol{V}) \quad \forall \boldsymbol{V} \in \mathscr{U}^{h} .
$$

To measure the effectiveness of the solution scheme, we distinguish whether an inhibited or non-inhibited problem is solved. When inhibited problems are considered, the solution using the above approach is effective as proven in [1]. However, when a non-inhibited problem is solved, the numerical phenomenon of "locking" occurs which severely reduces the rate of convergence. Namely, in this case the discrete variational problem considered is

Find $\boldsymbol{U}_{t}^{h} \in \mathscr{U}^{h}$ such that

$$
A\left(\boldsymbol{U}_{t}^{h}, \boldsymbol{V}\right)+\frac{1}{t^{2}} D\left(\boldsymbol{U}_{t}^{h}, \boldsymbol{V}\right)=F_{b}(\boldsymbol{V}) \quad \forall \boldsymbol{V} \in \mathscr{U}^{h},
$$

and the convergence is highly influenced by how rich $\mathscr{U}^{h}$ is in $\mathscr{U}_{0}$. In the worst case $\mathscr{U}^{h} \cap \mathscr{U}_{0}=\{\boldsymbol{0}\}$ leading to total loss of convergence

$\lim _{t \rightarrow 0} \boldsymbol{U}_{t}^{h}=\mathbf{0} \neq \lim _{t \rightarrow 0} \boldsymbol{U}_{t}=\boldsymbol{U}_{0}^{b}$.

In practice, extremely fine meshes are needed for an accurate solution when $t$ is small, and the displacement-based finite element procedure becomes unpractical. The remedy is to use an appropriate mixed formulation. The aim with this approach is to interpolate displacements and strains (or stresses) in such a manner as to have no locking of the discretization in the non-inhibited case for any value of thickness $t$, and to have, as well, a uniformly good behavior for the membrane-dominated case. Ideally, the rate of convergence would be optimal, independent of whether a bending-dominated or membrane-dominated problem is considered and independent of the shell thickness.

The key to reaching this optimal behavior is to use an appropriate mixed method with the appropriate "well-balanced" interpolations. The general mixed method that provides the basis of the MITC shell el- ements is

Find $\boldsymbol{U}^{h}=\left(\boldsymbol{u}^{h}, \boldsymbol{\beta}^{h}\right) \in \mathscr{U}^{h}=\left(\mathscr{V}^{h}, \mathscr{B}^{h}\right)$

and

$$
\begin{aligned}
& \boldsymbol{E}^{h}=\left\{\varepsilon_{i j}^{h}\right\} \in \mathscr{E}^{h} \text { such that } \\
& \left\{\begin{array}{l}
t^{3} \tilde{A}\left(\boldsymbol{E}^{h}, \boldsymbol{\varepsilon}(\boldsymbol{V})\right)+t \tilde{D}\left(\boldsymbol{E}^{h}, \boldsymbol{\varepsilon}(\boldsymbol{V})\right)=G(\boldsymbol{V}) \\
\tilde{A}\left(\boldsymbol{E}^{h}-\boldsymbol{\varepsilon}\left(\boldsymbol{U}^{h}\right), \boldsymbol{\Psi}\right)=0 \\
\tilde{D}\left(\boldsymbol{E}^{h}-\boldsymbol{\varepsilon}\left(\boldsymbol{U}^{h}\right), \boldsymbol{\Psi}\right)=0 \quad \forall \boldsymbol{V} \in \mathscr{U}^{h}, \boldsymbol{\Psi}=\left\{\psi_{i j}\right\} \in \mathscr{E}^{h},
\end{array}\right.
\end{aligned}
$$

where

$\mathscr{E}^{h}=\left\{\boldsymbol{\Psi}=\left\{\psi_{i j}\right\}, \psi_{i j} \in \mathscr{E}_{i j} h\right.$,

and

$\tilde{A}(\boldsymbol{\varepsilon}(\boldsymbol{U}), \boldsymbol{\varepsilon}(\boldsymbol{V}))=A(\boldsymbol{U}, \boldsymbol{V})$

$\tilde{D}(\boldsymbol{\varepsilon}(\boldsymbol{U}), \boldsymbol{\varepsilon}(\boldsymbol{V}))=D(\boldsymbol{U}, \boldsymbol{V})$.

Here $\mathscr{E}^{h}$ is the subspace of assumed strains and includes assumed bending, membrane and shear strains. The displacement and strain interpolations of the elements are summarized in Appendix A. The formulation can be derived from the Hellinger-Reissner variational principle (and therefore, also from the $\mathrm{Hu}-\mathrm{Washizu}$ variational principle), see Appendix B. However, a stability and convergence analysis is necessary to assess whether the actual discretization is effective. This analysis is difficult to perform analytically for a general setting, therefore we resort to numerical assessments. For the non-inhibited case, we would like that the inf-sup condition be satisfied for the selected interpolations. The results of our inf-sup condition study are presented in Ref. [7] and show that a numerical inf-sup test is satisfied. Our objective in the following section is to give the results obtained in convergence studies.

\section{Numerical convergence studies}

The test problems we use for the convergence studies are described in Ref. [1], where the reasons for selecting these problems are also given. Using these problems (summarized below), we proceed in each case as follows.

For each of the test problems, we run a sequence of meshes and depict the convergence of the relative error in strain energy of the approximation $E_{\mathrm{r}}$ versus a mesh density indicator $N$, where 

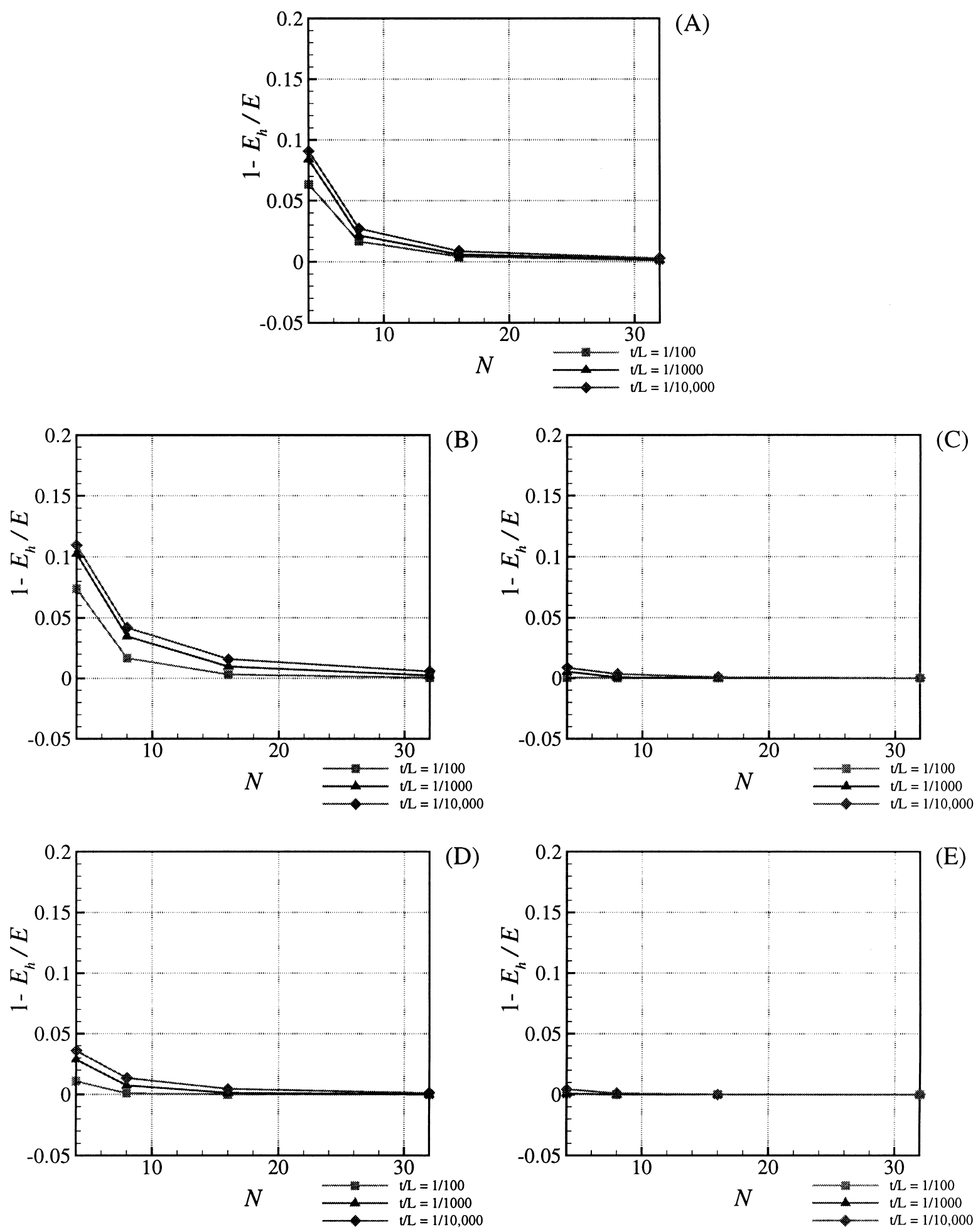

Fig. 4. Convergence in strain energy for the clamped cylinder problem. Uniform meshes. (A) MITC4 element; (B) QUAD9; (C) MITC9; (D) QUAD16; (E) MITC16; (F) TRI6; (G) MITC6. 

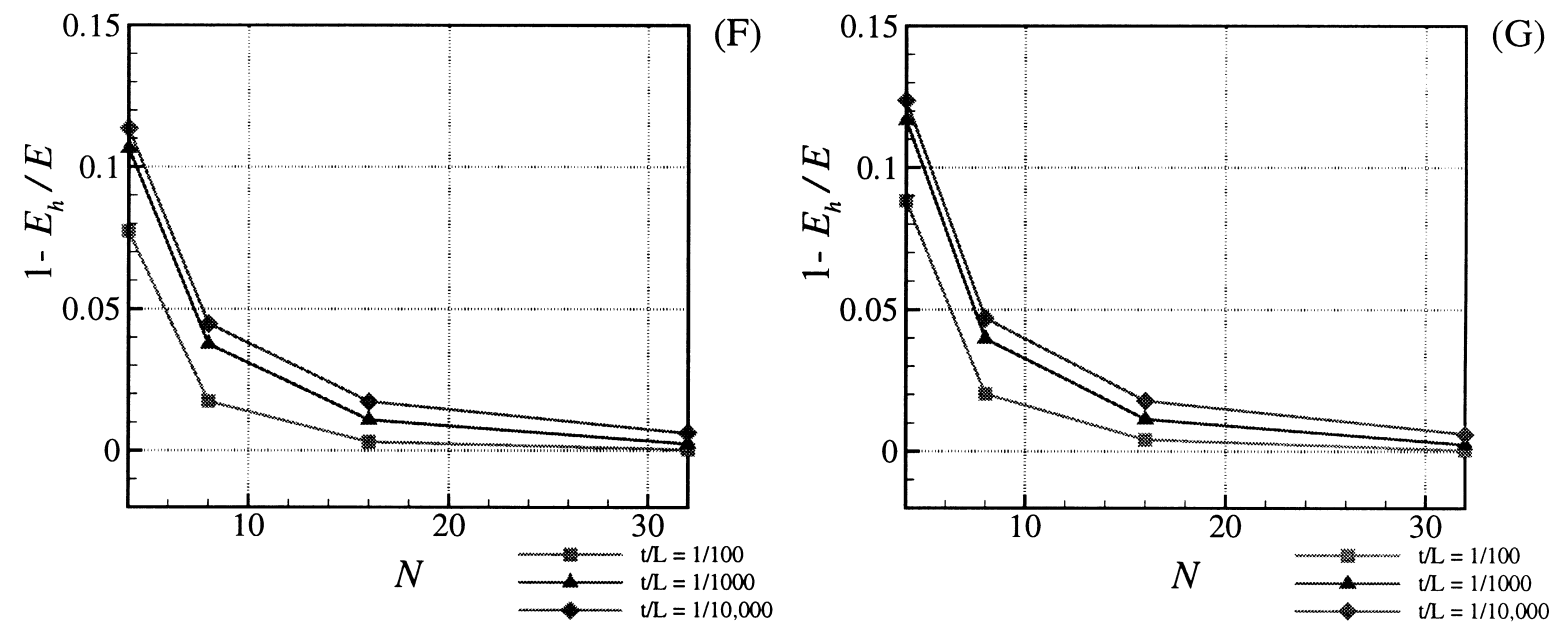

Fig. 4 (continued)

$E_{\mathrm{r}}=\frac{E-E_{h}}{E}$,

with $E_{h}$ being the strain energy of the finite element approximation and $E$ being the strain energy calculated using either the exact solution of the mathematical model, or, when no analytical solution is available, other finite element schemes or very fine meshes of high-order shell elements.

In addition, we plot the $E_{\mathrm{r}}$ values versus the element size $h$ in the logarithmic scale to estimate the convergence constant $c$ and order of convergence $\hat{k}$ of the convergence equation:

$\left|E_{\mathrm{r}}\right|=c h^{2 \hat{k}}$

The numerical simulations are repeated for a number of thickness parameter values, and changes in the convergence properties of the element are reflected in changes of the convergence constant $c$ and order of convergence $\hat{k}$.

Ideally, we would like to have an element with $\hat{k}$ independent of the nature of the problem (i.e., bending- or membrane-dominated) and the thickness $t$. In addition, the convergence constant $c$ should exhibit no dependence on $t$ for each problem under consideration. Moreover, the order of convergence $\hat{k}$ should be optimal. In general, the optimal order of convergence $k$ is prescribed by the order of the discretization scheme under consideration (and the physics of the problem,

\footnotetext{
${ }^{1}$ The values for displacements and deformation energy in Ref. [8] should be multiplied by $\left(1-v^{2}\right)$ and $\left(1-v^{2}\right)^{2}$, respectively.
}

smoothness of the solution, presence of stress concentrations due to loading, boundary layers, etc.) [2].

\subsection{Membrane-dominated tests}

\subsubsection{Clamped cylindrical shell}

We consider a cylindrical shell of uniform thickness $t$, length $2 L$ and radius $R$ loaded by an axially-constant pressure distribution $p(\varphi)$ acting on the outer surface of the shell,

$p(\varphi)=P_{0} \cos (2 \varphi)$.

At a point $P$ on the cylinder's midsurface, we select the axial displacement $u$, tangential displacement $v$, radial displacement $w$, rotation of the normal about the $X$-axis $\beta_{1}$, and rotation of the normal about the tangent vector $\beta_{2}$ as the displacement variables (see Fig. 1).

By symmetry, we can limit calculations to the shaded region $\mathrm{ABCD}$ with the following symmetry and boundary conditions imposed:

$u=w=0$ along $\mathrm{AB} ;$

$u=\beta_{2}=0$ along $\mathrm{BC} ; v=\beta_{1}=0$ along $\mathrm{CD} ;$

$u=v=w=\beta_{1}=\beta_{2}=0$ along $\mathrm{AD}$.

The analytical solution for the problem using the Naghdi shell theory has been published for any given value of $t\left(\right.$ see $\left.[8]^{1}\right)$. The problem is membrane-dominated with the combined bending and shear energy being less than $2 \%$ even for a moderately thick case of 

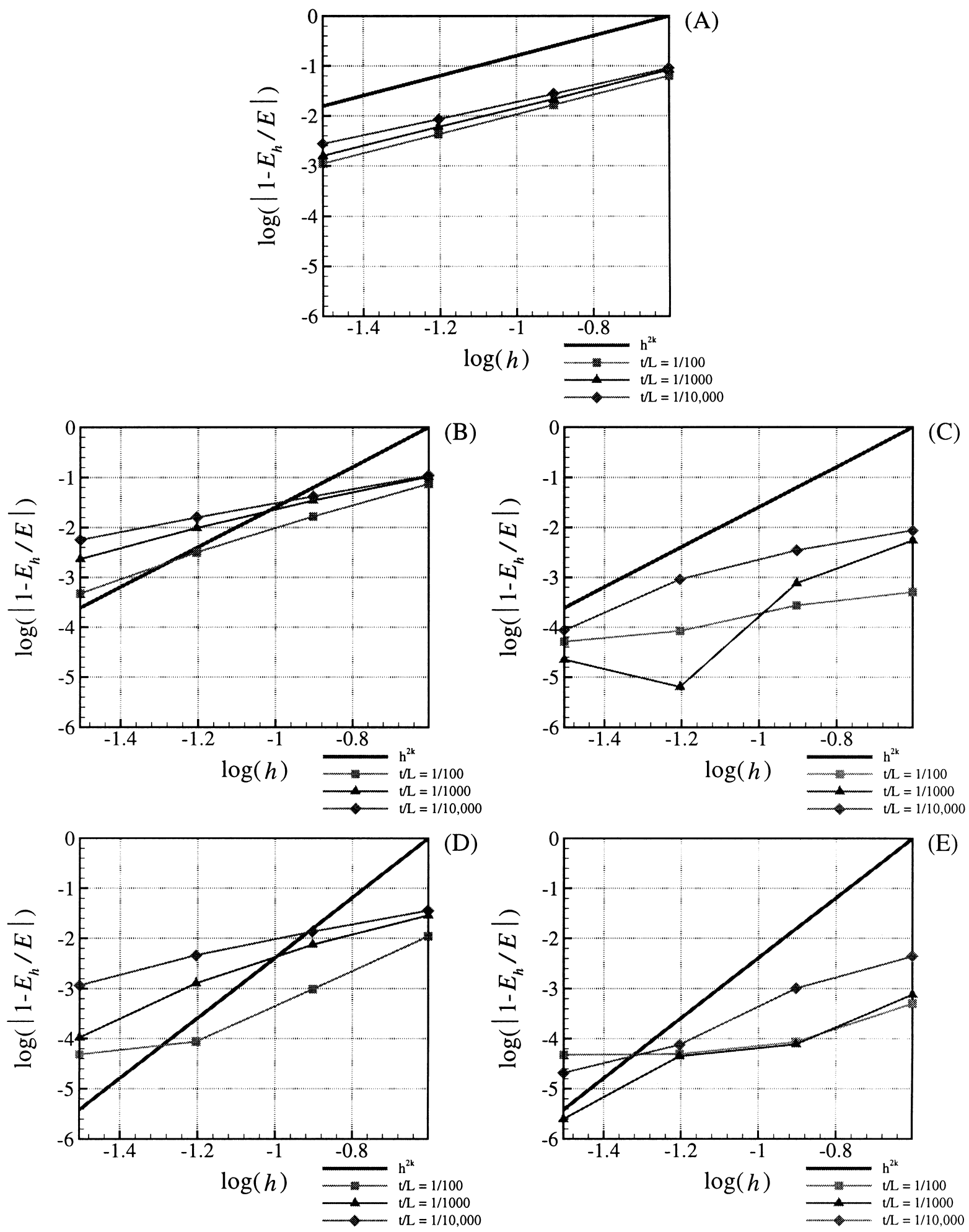

Fig. 5. Convergence in strain energy for the clamped cylinder problem. Uniform meshes. (A) MITC4 element; (B) QUAD9; (C) MITC9; (D) QUAD16; (E) MITC16; (F) TRI6; (G) MITC6. 

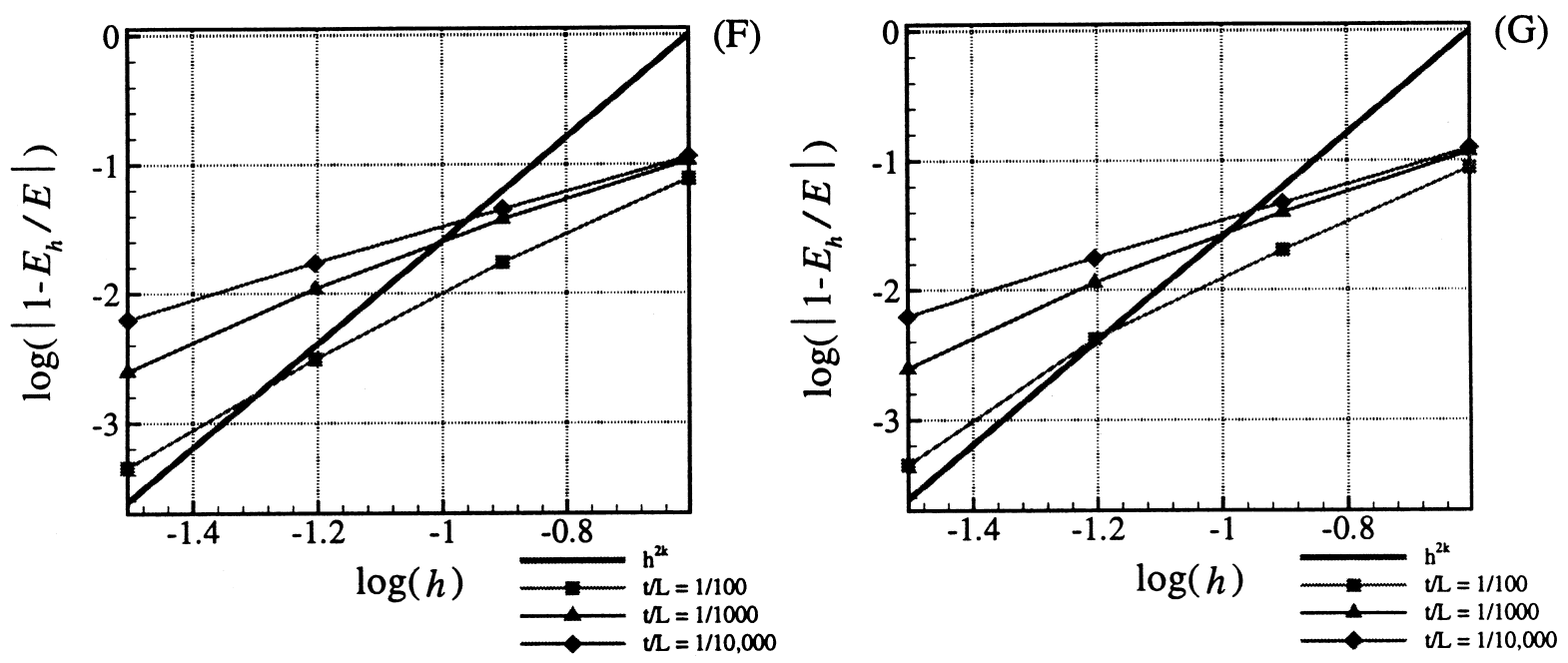

Fig. 5 (continued)

$t / L=1 / 100$ (see Fig. 2) and hence we scale the applied loading with thickness $t$ as

$$
P_{0}=p_{0} t
$$

where $p_{0}$ is a constant independent of $t$.

First we consider uniform $N \times N$ meshes with element sides aligned with the principal directions of curvature, where $N$ is the number of subdivisions per side of the discretized domain (in our tests $N=4,8$,

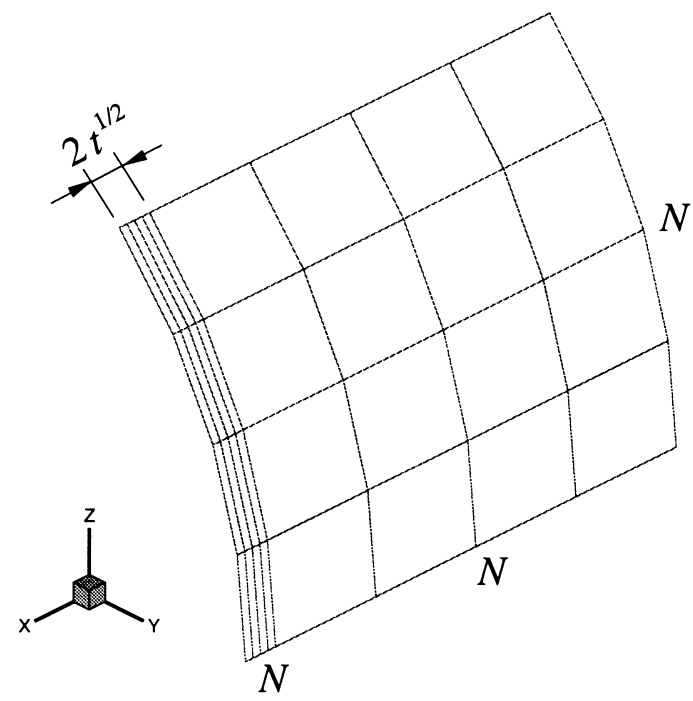

Fig. 6. A mesh grading scheme for the cylindrical shell problem, mesh density $N=4$.
16 and 32). This sequence of meshes is repeated for each tested element for values of dimensionless thickness parameter $t / L$ ranging from $1 / 100$ to $1 / 10,000$.

Fig. 3 gives a comparison of the finite element results with the analytical solution for the radial displacement profile calculated using the elements of the MITC family and the 9- and 16-node displacementbased general shell elements (QUAD9 and QUAD16) for the case of $t / L=1 / 10,000$.

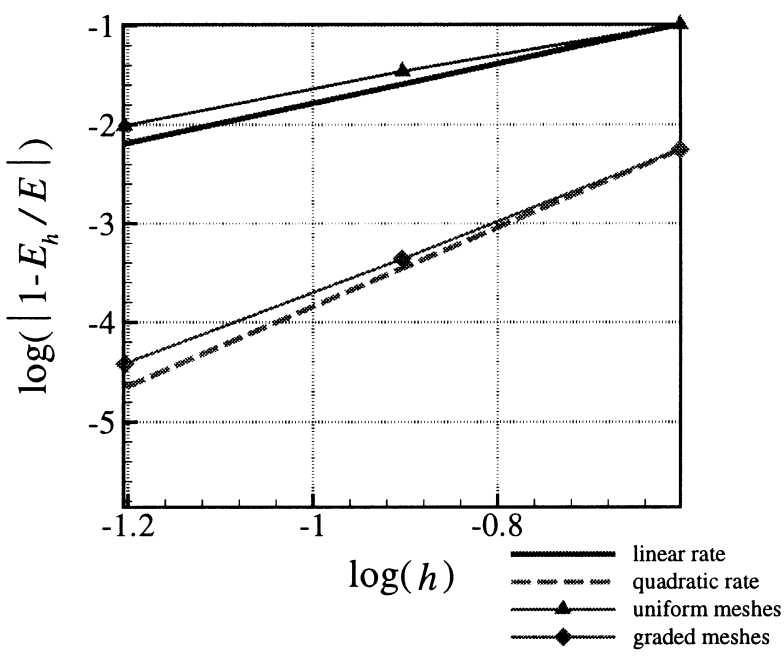

Fig. 7. Convergence in strain energy for the clamped cylinder problem using the nine-node displacement-based element QUAD9 in uniform and graded meshes for the case $t / L=1 / 1000$. 
Table 1

Benchmark values for the strain energy $E$ and vertical displacement $w$ at point $x=0, y=0$ for the hemispherical cap under axisymmetric pressure. We use $R=1 \mathrm{~m} ; E=2 \times 10^{5}$ $\mathrm{MPa} ; v=0.3 ; p_{0}=1 \mathrm{MPa}$

\begin{tabular}{lll}
\hline$t / R$ & $10241 \mathrm{D}$ axisymmetric three-node shell elements \\
\cline { 2 - 3 } & Strain energy $E(\mathrm{~N} \cdot \mathrm{mm})$ & $w(0,0)(\mathrm{mm})$ \\
\hline $1 / 100$ & $6.79244 \times 10^{-5}$ & $-7.73688 \times 10^{-3}$ \\
$1 / 1000$ & $7.62474 \times 10^{-6}$ & $-8.08931 \times 10^{-3}$ \\
$1 / 10,000$ & $7.88885 \times 10^{-7}$ & $-8.19934 \times 10^{-3}$ \\
\hline
\end{tabular}

All the elements perform quite well. However, some numerical instabilities especially pronounced for the displacement-based elements can be observed at the clamped end. These instabilities are due to the presence of boundary layers and can be eliminated using graded meshes (as derived in Ref. [8], boundary layers play a dominant role in a $\sim 2 \sqrt{t}$ region at the fixed end).

Figs. 4 and 5 give the convergence in relative error $E_{\mathrm{r}}$ for the problem under consideration for a sequence of $t / L$ values. The six-node displacement-based element is referred to as the TRI6 element.

The convergence curves of the MITC4 element stay virtually unaffected by changes in thickness $t$, and the element's order of convergence $\hat{k}$ is very close to its theoretical value of $k=1$.

The convergence curves for the other MITC elements have noticeably lower convergence constants $c$ than their displacement-based counterparts and reach the accuracy of $1.0 \%$ with the coarsest mesh. Note that for the case of $t / L=1 / 100$ the elements do not converge further after reaching the accuracy level of $\sim 0.01 \%$, which is explained by differences

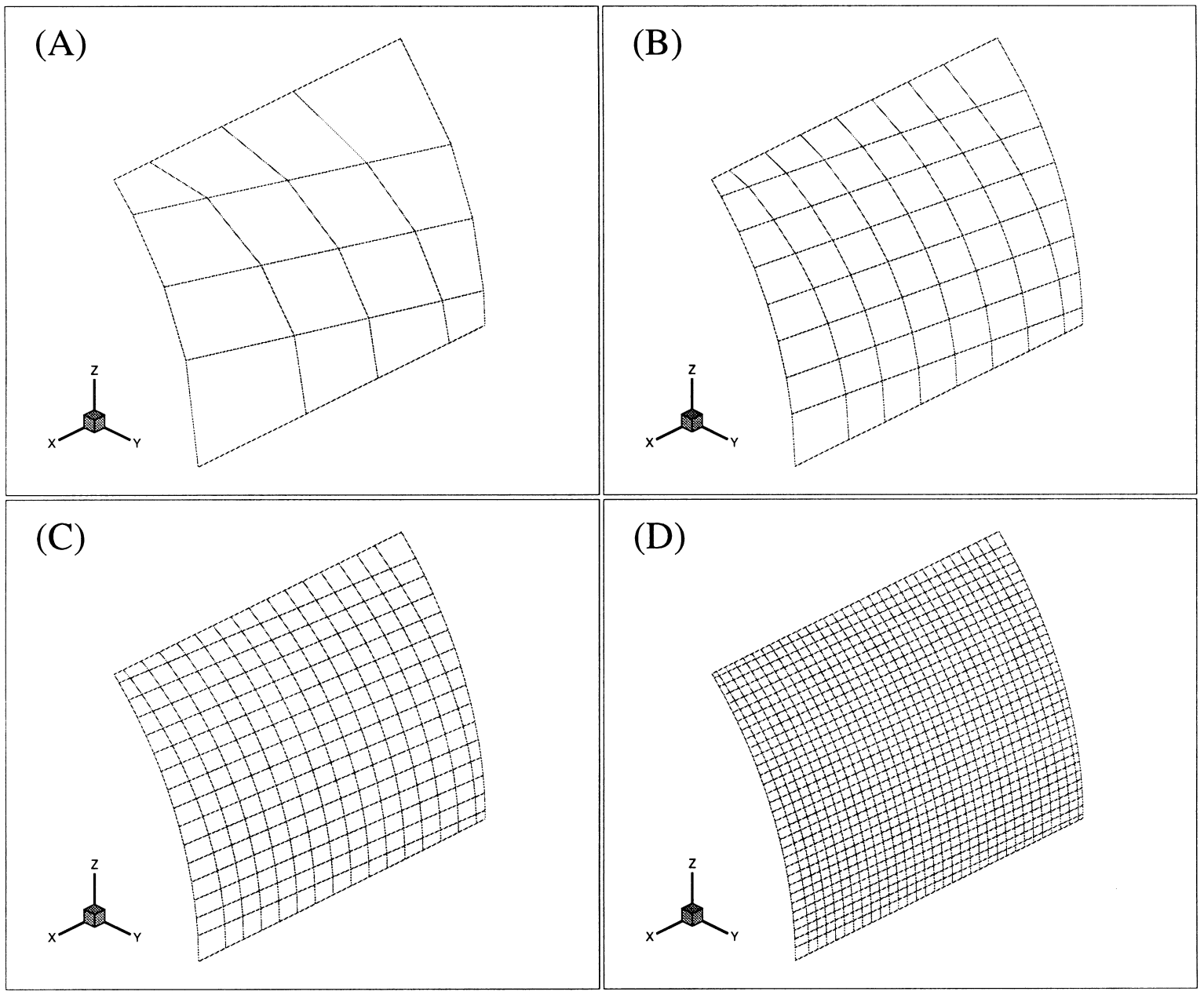

Fig. 8. A sequence of distorted meshes used for cylindrical shell. (A) The $4 \times 4$ mesh; (B) $8 \times 8$; (C) $16 \times 16$; (D) $32 \times 32$. 

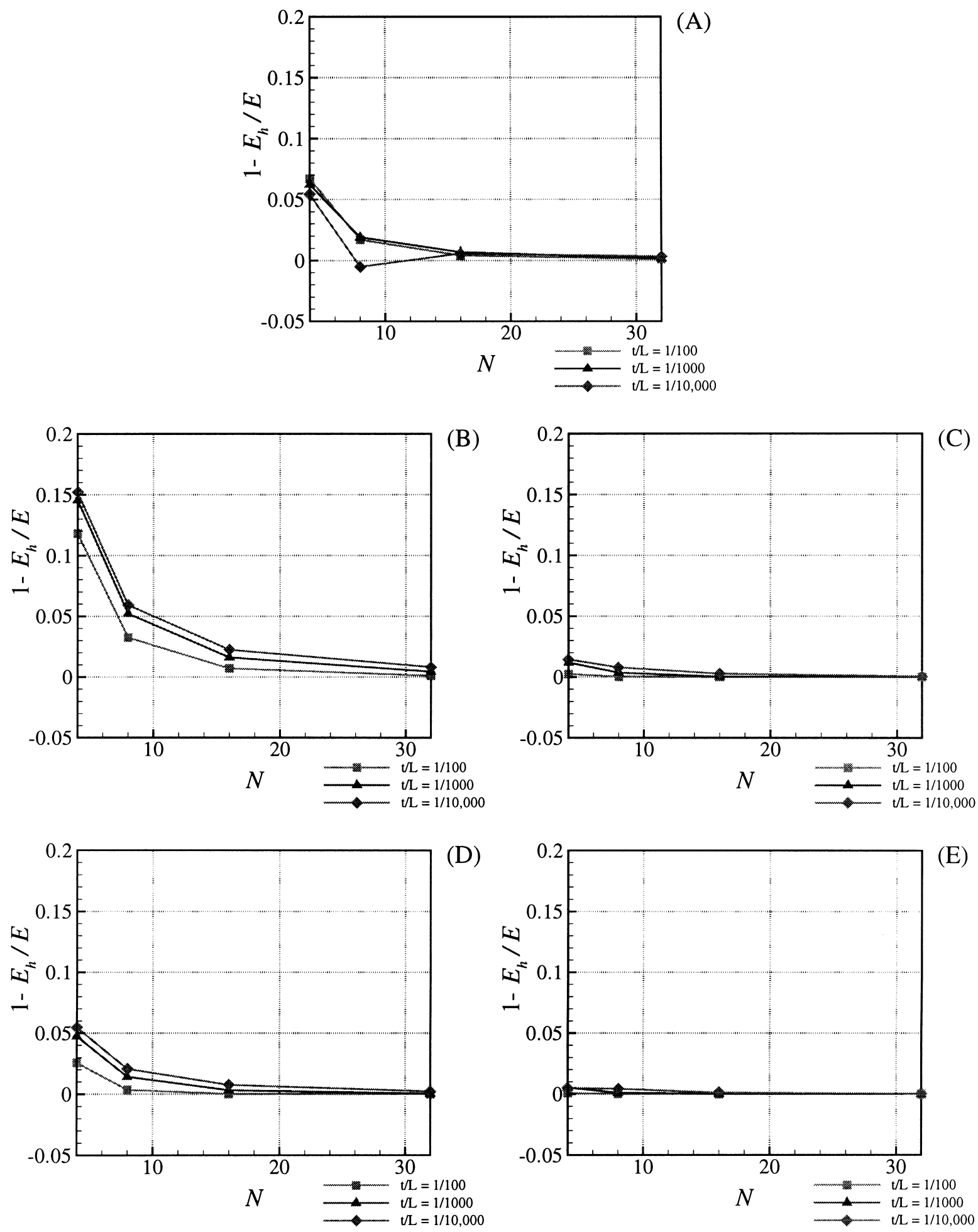

Fig. 9. Convergence in strain energy for the clamped cylinder problem. Distorted meshes. (A) MITC4 element; (B) QUAD9; (C) MITC9; (D) QUAD16; (E) MITC16. 

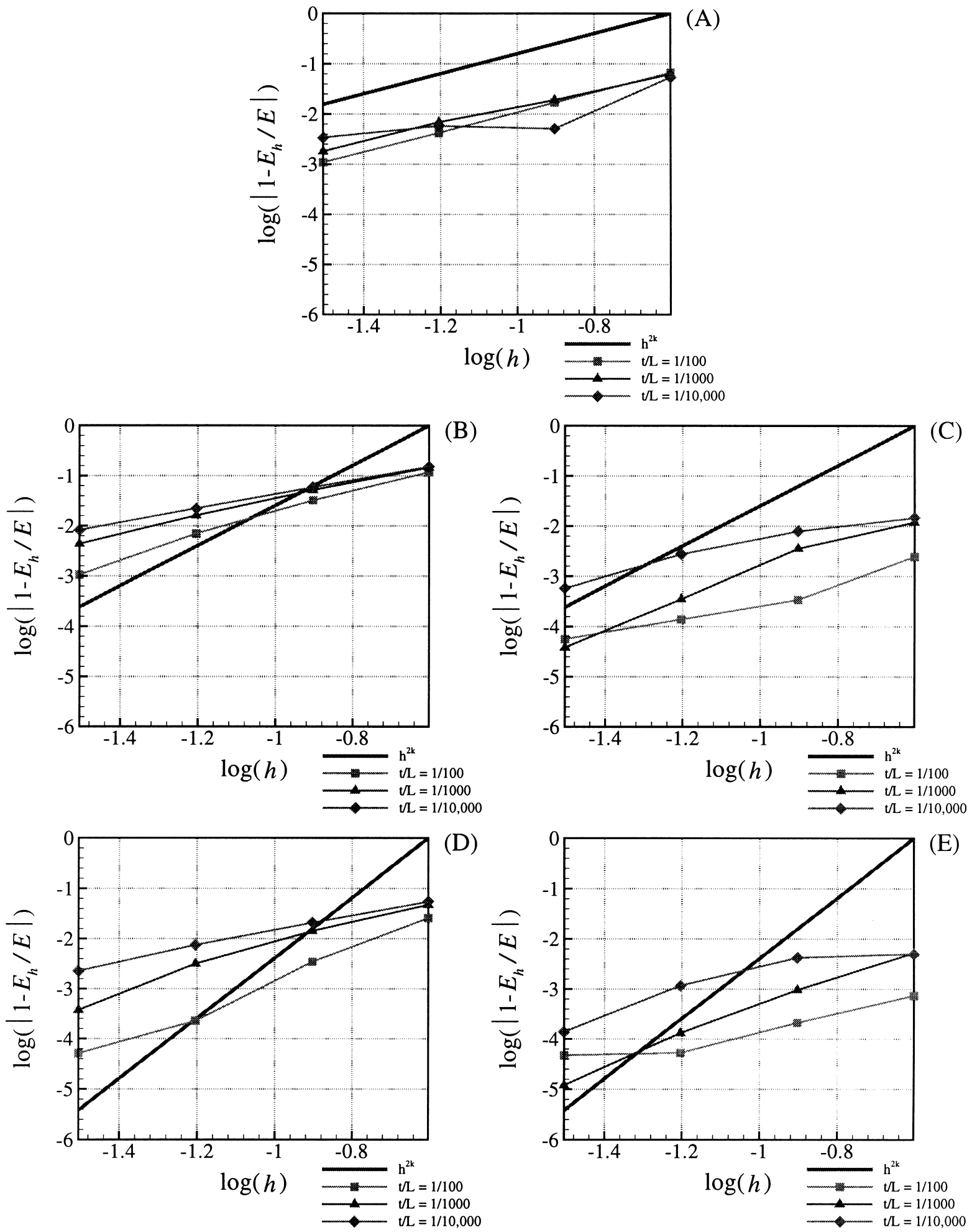

Fig. 10. Convergence in strain energy for the clamped cylinder problem. Distorted meshes. (A) MITC4 element; (B) QUAD9; (C) MITC9; (D) QUAD16; (E) MITC16. 


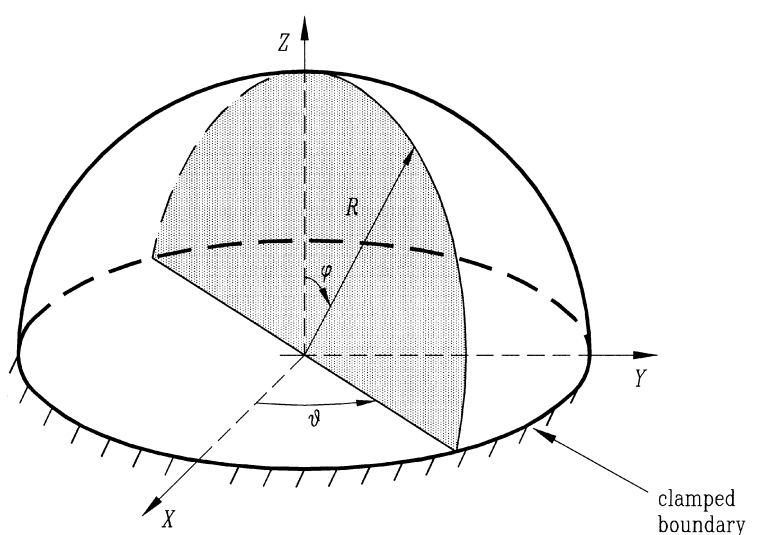

Fig. 11. Clamped hemispherical cap. between the reference (Naghdi theory) solution and the solution to our underlying shell model [6].

As pointed out in [8], the fact that the observed convergence rates are lower than the respective "optimal" rates (equal to the order of the polynomial interpolations in the elements) may be due to the presence of stress concentration in the boundary layer region and can be improved using mesh grading. To identify the effect of mesh grading, we consider the following mesh grading scheme: the discretized domain is separated into two regions - the boundary layer region of width $2 \sqrt{t}$ in the $X$-direction, and the smooth solution region of width equal to $1-2 \sqrt{t}$. Both regions are then meshed with the equal mesh density $N$ as shown in Fig. 6.

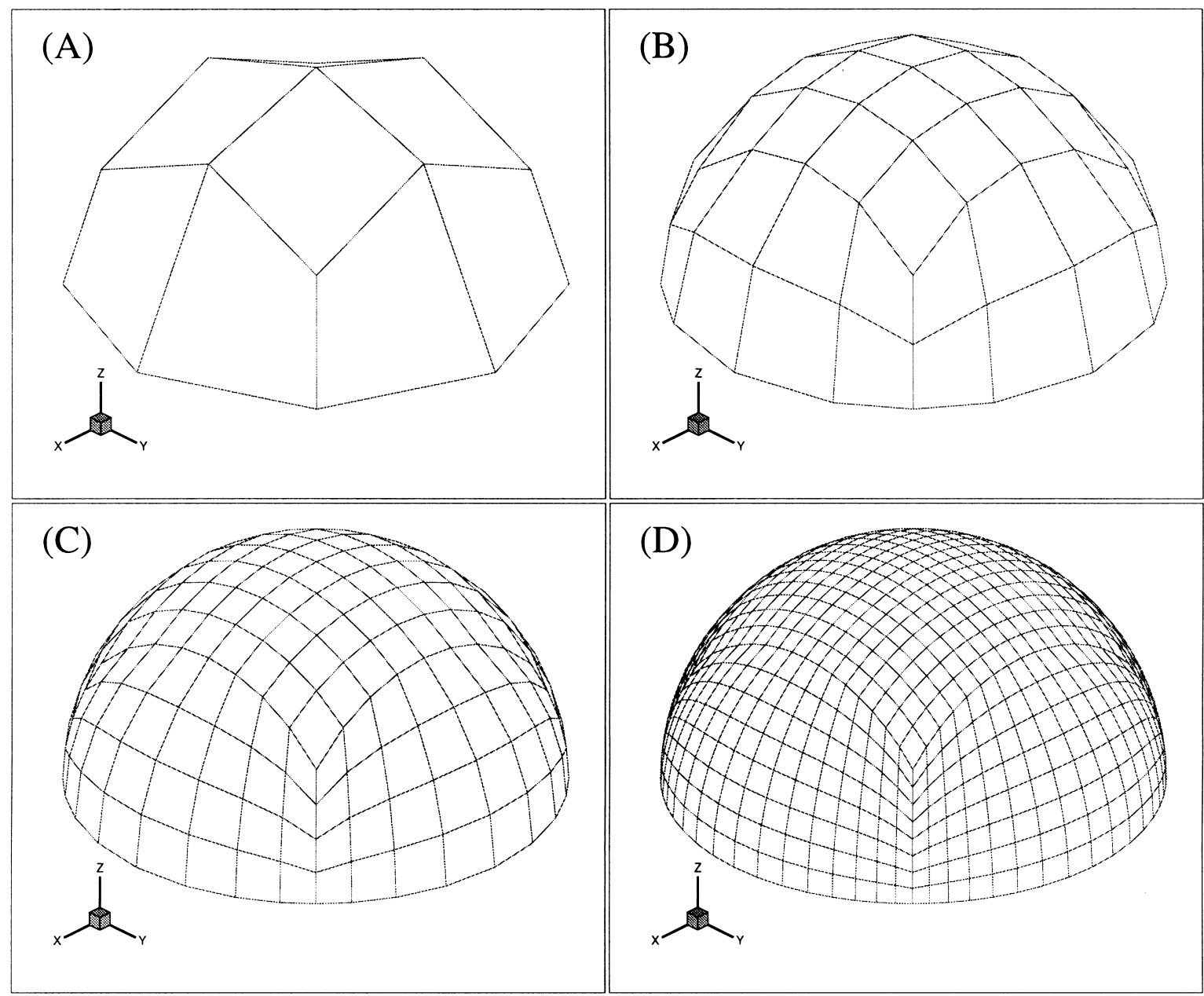

Fig. 12. A sequence of $N_{\varphi} \times N_{\vartheta}$ meshes of quadrilateral elements for the hemispherical cap problem. (A) The $2 \times 8$ mesh; (B) $4 \times 16$; (C) $8 \times 32$; (D) $16 \times 64$. 

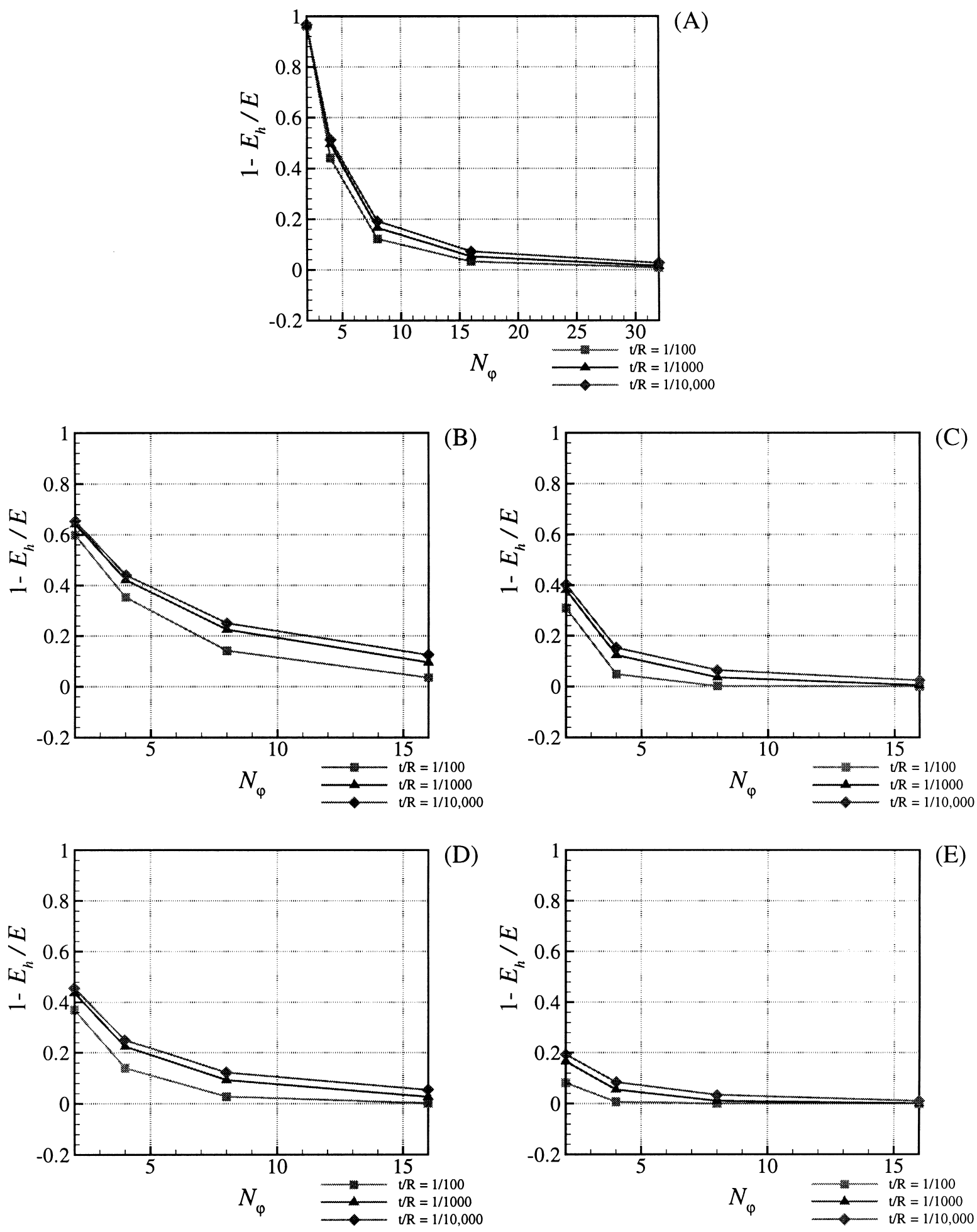

Fig. 13. Convergence in strain energy for the clamped hemispherical cap problem. (A) MITC4 element; (B) QUAD9; (C) MITC9; (D) QUAD16; (E) MITC16; (F) TRI6; (G) MITC6. 



Fig. 13 (continued)

Fig. 7 illustrates the change in the convergence rate for the nine-node displacement-based element QUAD9 when a sequence of graded meshes is considered for the case of $t / L=1 / 1000$. The order of convergence drastically increases and virtually reaches the optimal rate $k$, and a pronounceable reduction in the convergence constant $c$ further underlines the importance of mesh grading.

We also test the elements using distorted meshes in which element sides are not aligned with the principal directions of curvature. Such sequence is shown in Fig. 8.

Figs. 9 and 10 display convergence results calculated using the distorted meshes of Fig. 8. Overall, no dramatic changes in convergence can be observed, however, the characteristics have worsened for all elements. This effect can be partly attributed to poorer geometry approximation using distorted meshes.

\subsubsection{Clamped hemispherical cap}

We consider a hemisphere of radius $R$, fully clamped at $\varphi=\pi / 2$ (see Fig. 11), and uniform thickness $t$ loaded with an axisymmetric pressure distribution

$p(\varphi)=P_{0} \cos (2 \varphi)$.

The problem is membrane-dominated, hence we scale the applied load $p$ with $t$.

Since no analytical solution is available for this problem, we use a 1D axisymmetric shell finite element model to obtain benchmark values for the strain energy, see Table 1.

We use a sequence of $N_{\varphi} \times N_{\vartheta}$ meshes to study the convergence of the elements for $t / R$ ratios from $1 / 100$ to $1 / 10,000$, where $N_{\varphi}$ is the number of divisions in the $\varphi$-direction, and $N_{\vartheta}$ is the number of divisions in the $\vartheta$-direction at $\varphi=\pi / 2$ (see Fig. 12).

Figs. 13 and 14 report the results of the finite element simulations.

Overall, all the elements have a relatively high value of the convergence constant $c$ and low convergence rates because of severe mesh distortions (the element sides are far from being aligned with the lines of principal curvature) and poor geometry approximation, especially for coarser meshes.

Like for the clamped cylinder problem, the MITC4 element exhibits virtually no dependence on $t$. The better performance of the other MITC elements (compared to the displacement-based elements) is reflected in lower values of convergence constants.

\subsection{Bending-dominated tests}

\subsubsection{Free cylindrical shell}

The geometry and loading are the same as in the

\section{Table 2}

Reference values for the strain energy $E$ and vertical displacement $w$ at point $X=L / 2, Y=0$ for the hyperbolic paraboloid, calculated using a $48 \times 24$ mesh of MITC16 elements. We use $L=1 \mathrm{~m} ; E=2 \times 10^{5} \mathrm{MPa} ; v=0.3$; density $\rho=8000$ $\mathrm{kg} / \mathrm{m}^{3}$

\begin{tabular}{lll}
$t / L$ & Strain energy $E(\mathrm{~N} \cdot \mathrm{m})$ & Displacement $w(L / 2,0)(\mathrm{m})$ \\
\hline $1 / 100$ & $1.6790 \times 10^{-3}$ & $-9.3355 \times 10^{-5}$ \\
$1 / 1000$ & $1.1013 \times 10^{-2}$ & $-6.3941 \times 10^{-3}$ \\
$1 / 10,000$ & $8.9867 \times 10^{-2}$ & $-5.2988 \times 10^{-1}$ \\
\hline
\end{tabular}



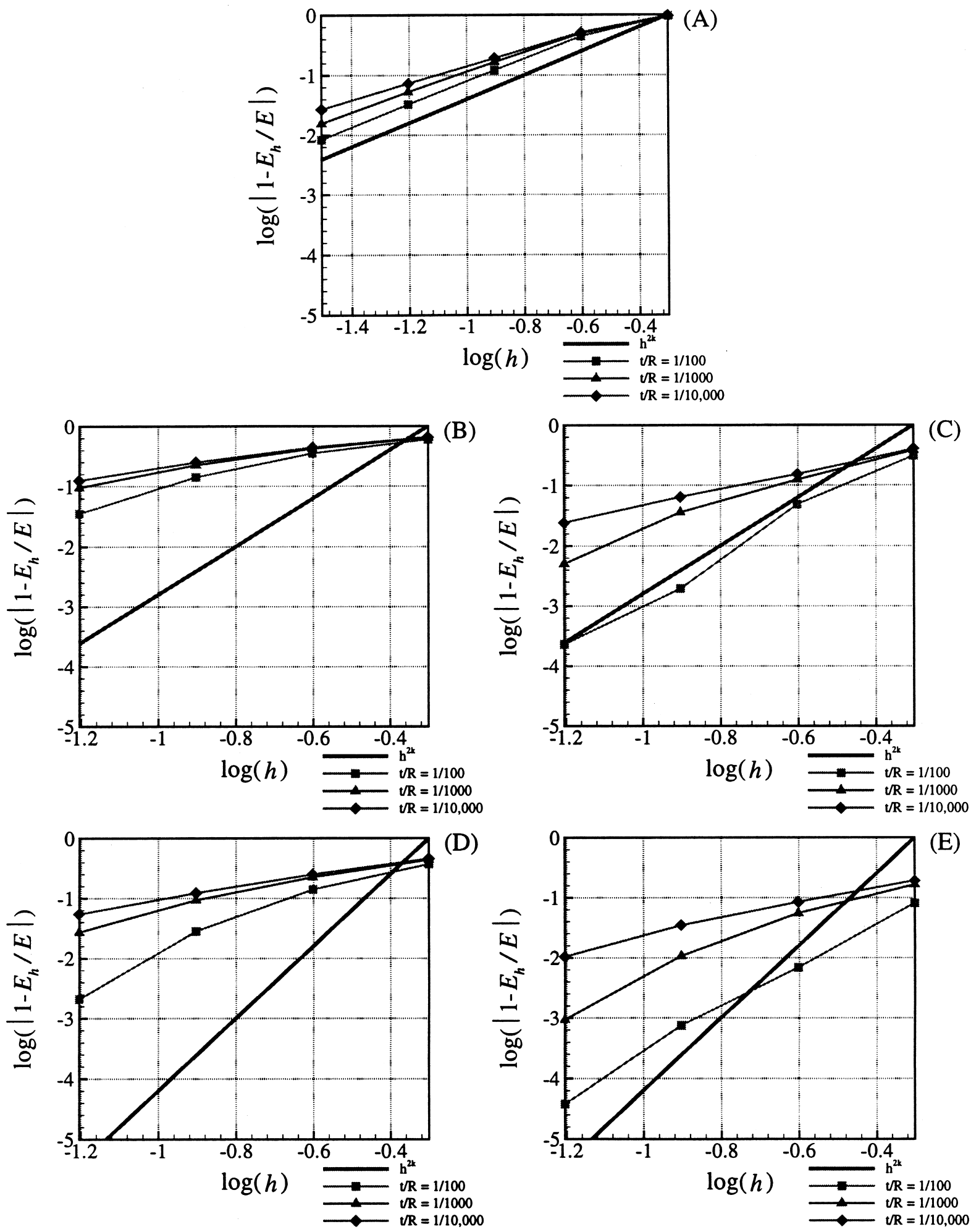

Fig. 14. Convergence in strain energy for the clamped hemispherical cap problem. (A) MITC4 element; (B) QUAD9; (C) MITC9; (D) QUAD16; (E) MITC16; (F) TRI6; (G) MITC6. 

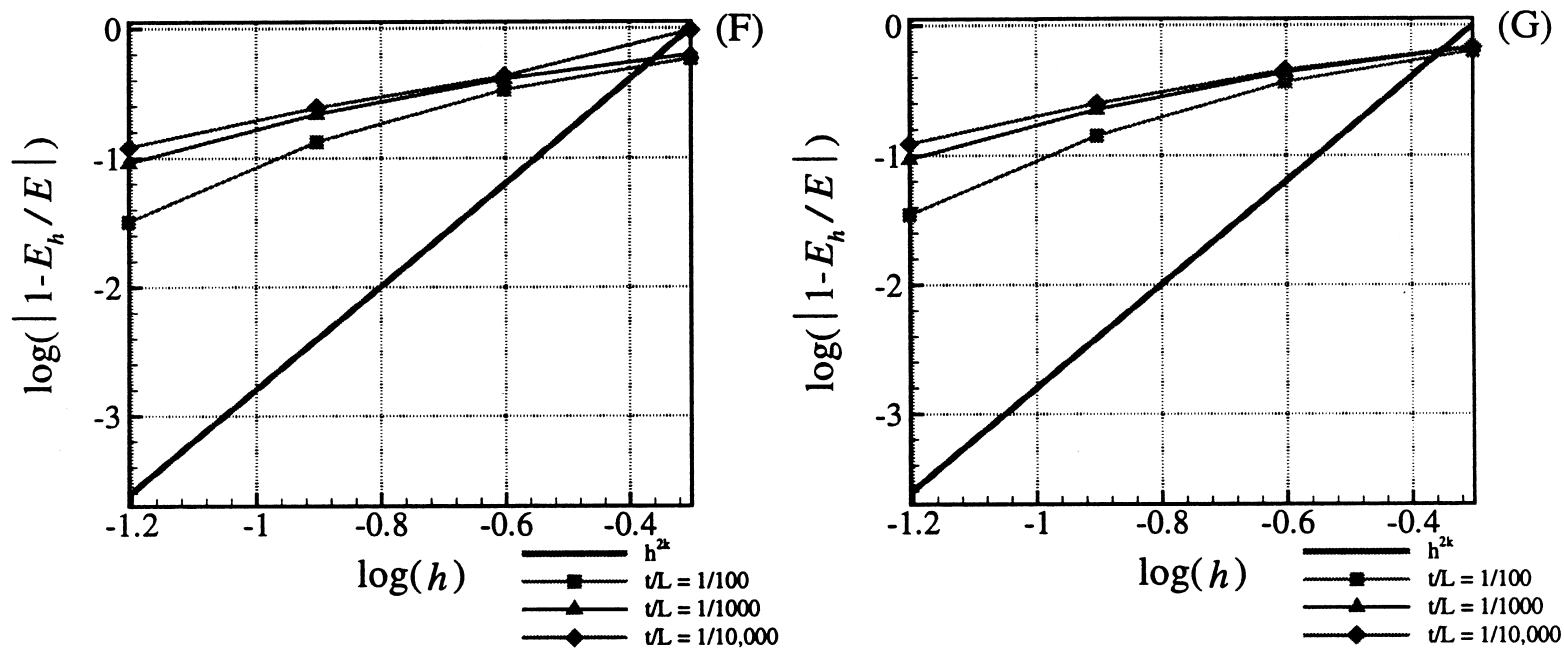

Fig. 14 (continued)

clamped cylinder problem considered above. The same boundary and symmetry conditions are applicable but we release all the fixities along the arc AD.

The bending energy prevails even for moderately large $t / L$ ratios - Fig. 15 shows that relative contributions of the shear and membrane terms are essentially negligible.

In the convergence study we use the Naghdi shell theory solution given in Ref. [8], where a general methodology of obtaining the exact solution for any given (positive) value of $t$ is demonstrated. Because bending is dominant in this problem, the load is rescaled as

$$
P_{0}=p_{0} t^{3}
$$

Fig. 16 presents a comparison of the analytical solution for the axial profile of the radial displacement $w$ with the finite element simulation results for the $t / L=$ $1 / 10,000$ case. The nine-node displacement-based element locks and results in zero displacements for coarser meshes. The QUAD16 element starts off quite far from the analytical solution but reaches decent accuracy for finer meshes. The MITC4 element displays robust convergence, and the higher-order MITC elements produce accurate results even for relatively coarse meshes.

The convergence in strain energy using uniform meshes is displayed in Figs. 17 and $18^{2}$. As in the

\footnotetext{
${ }^{2}$ Symbols " $*$ " on convergence graphs hereafter indicate that the numerical value of the associated point on the graph might be affected by computational (round-off) errors due to ill-conditioning of the stiffness matrix of the system.
}

clamped cylinder case, the MITC4 element convergence curves are "perfect" - they stay parallel to the optimal convergence line, and neither the convergence constant $c$ nor the order $\hat{k}$ are affected by variations in thickness.

The locking of the nine-node displacement-based element can be directly observed - its convergence curves flatten out as $t$ is decreased, and the relative error stays above the $70 \%$ level even for very fine meshes when $t / L=1 / 10,000$.

The behavior of the QUAD16 element is slightly affected by changes in thickness - the convergence constant of the element gets larger, indicating potential difficulties as $t$ is decreased.

The higher-order MITC elements converge to the accuracy of $0.1 \%$ in strain energy with the coarsest

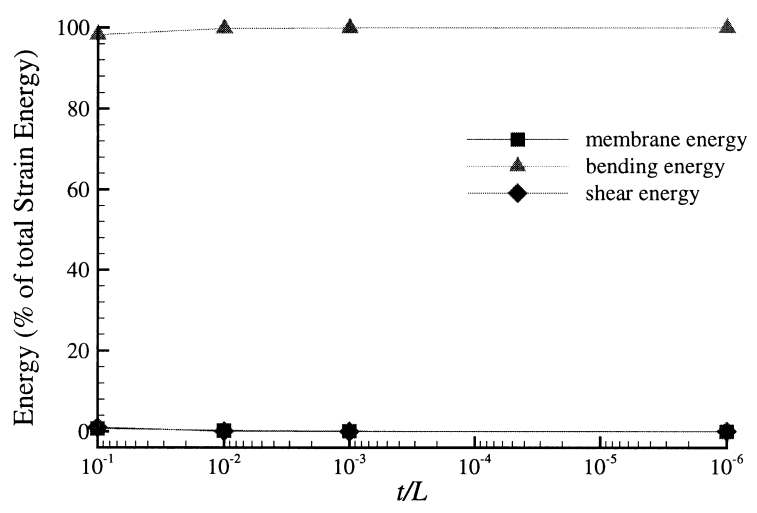

Fig. 15. Distribution of strain energy as a function of $t / L$ for the free cylindrical shell. 




(B)
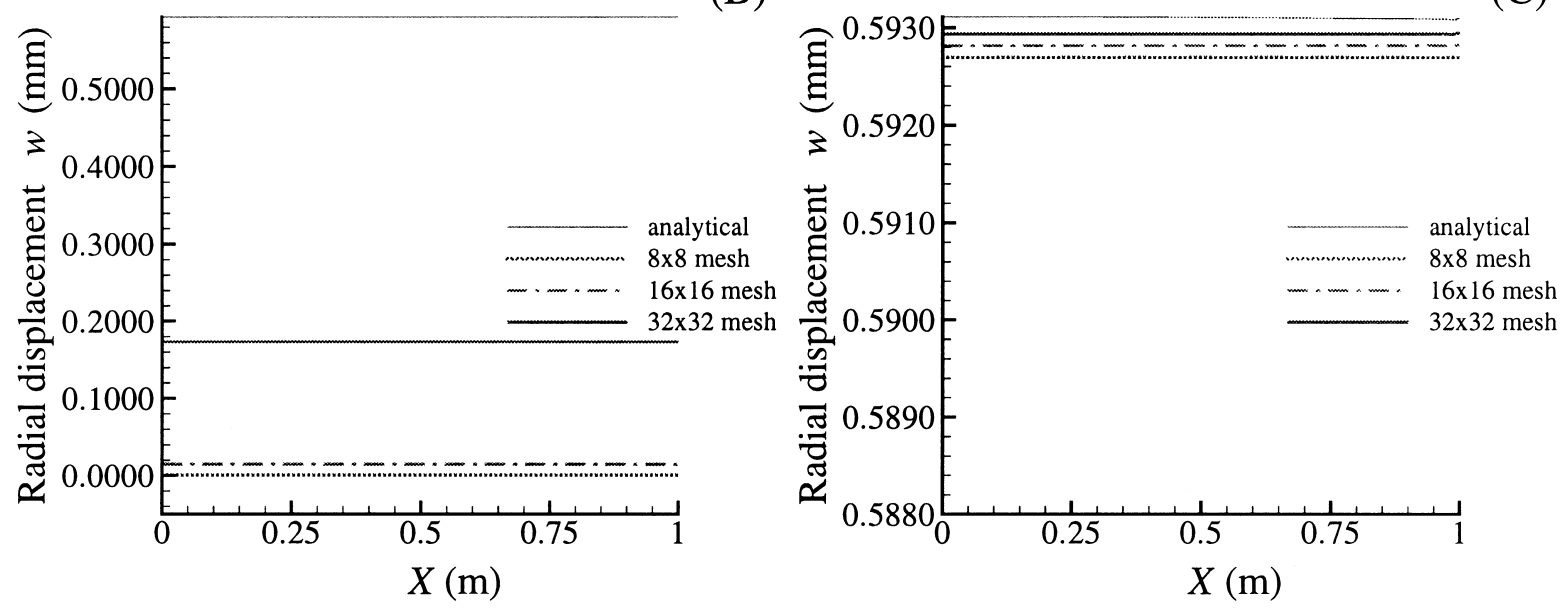

(D)

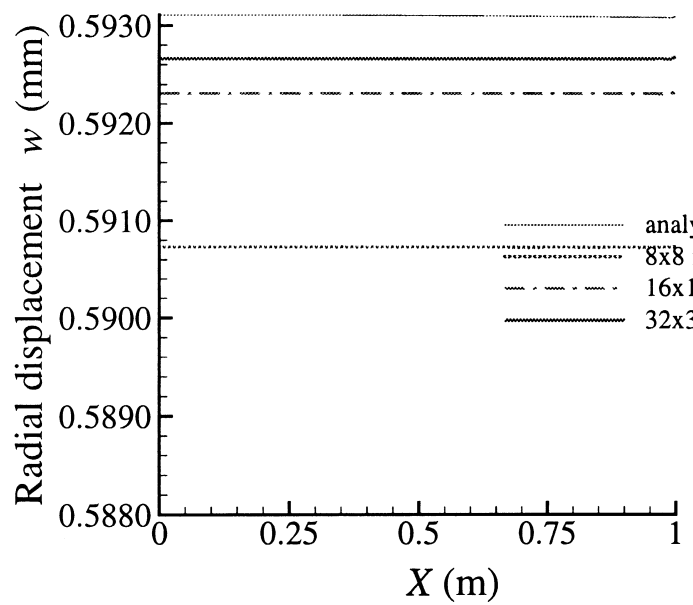

(E)

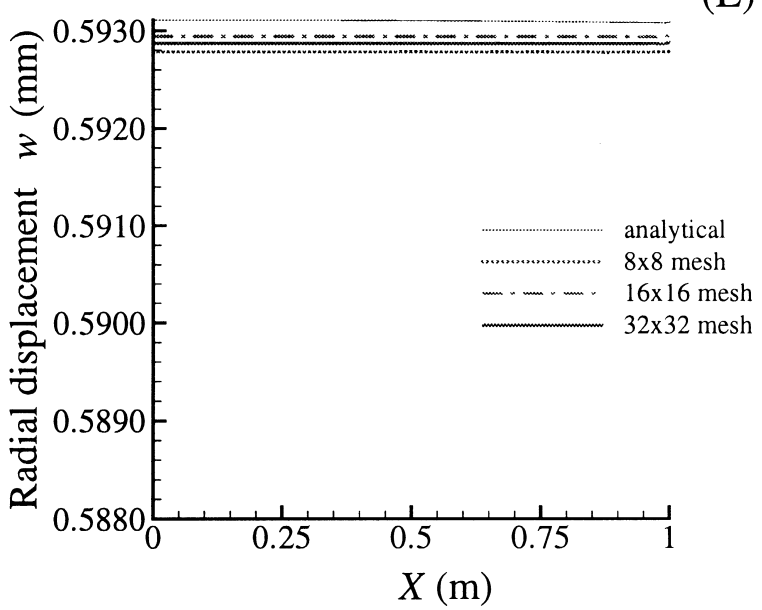

Fig. 16. Axial profile of radial displacement $w$ calculated along line $\mathrm{CD}$ for the free cylinder problem using $L=R=1 \mathrm{~m}$; $t / L=1 / 10,000 ; E=2 \times 10^{5} \mathrm{MPa} ; v=1 / 3 ; p_{0}=1 \mathrm{MPa}$. (A) MITC4 element; (B) QUAD9; (C) MITC9; (D) QUAD16; (E) MITC16. 

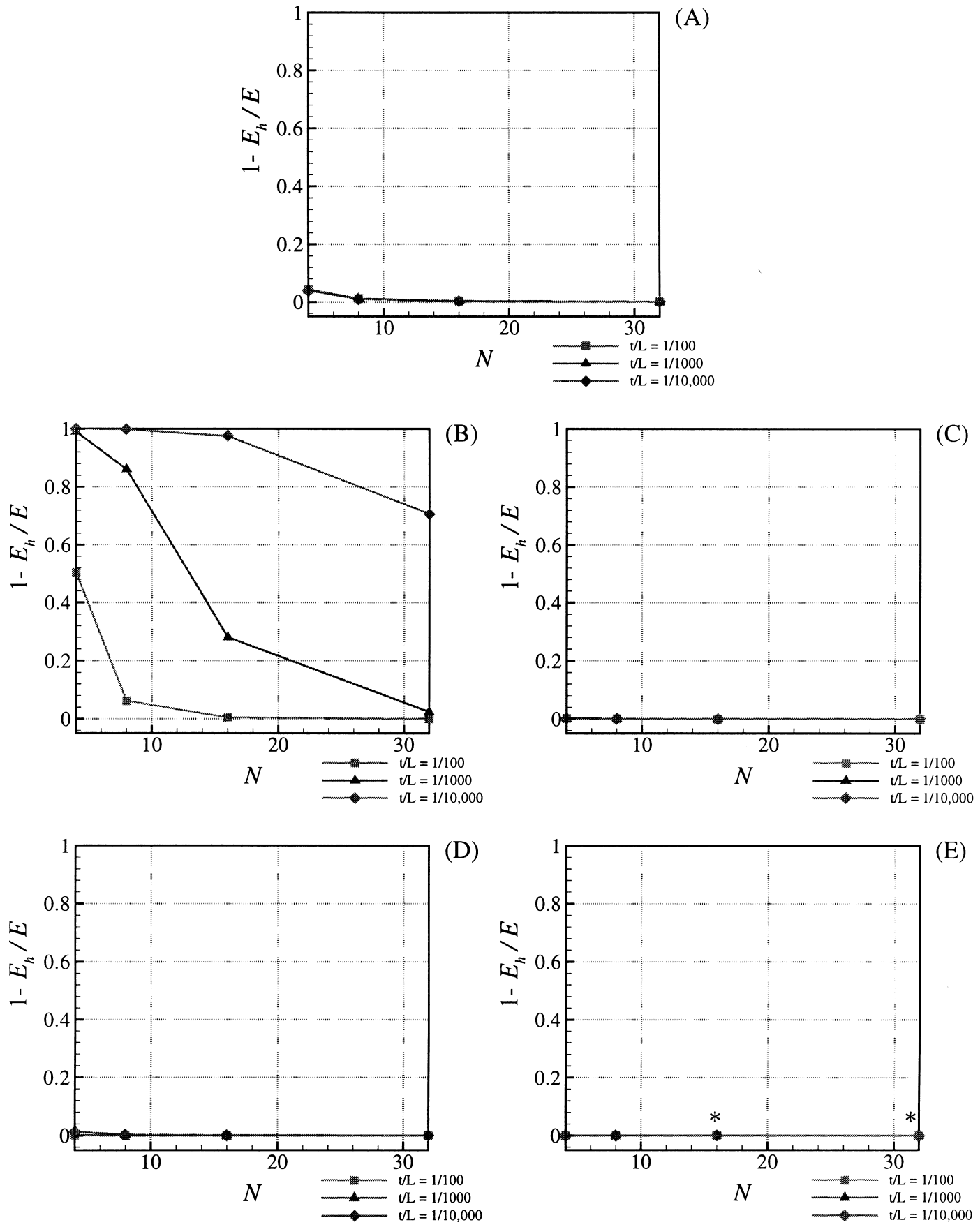

Fig. 17. Convergence in strain energy for the free cylinder problem. Uniform meshes. (A) MITC4 element; (B) QUAD9; (C) MITC9; (D) QUAD16; (E) MITC16; (F) TRI6; (G) MITC6. 

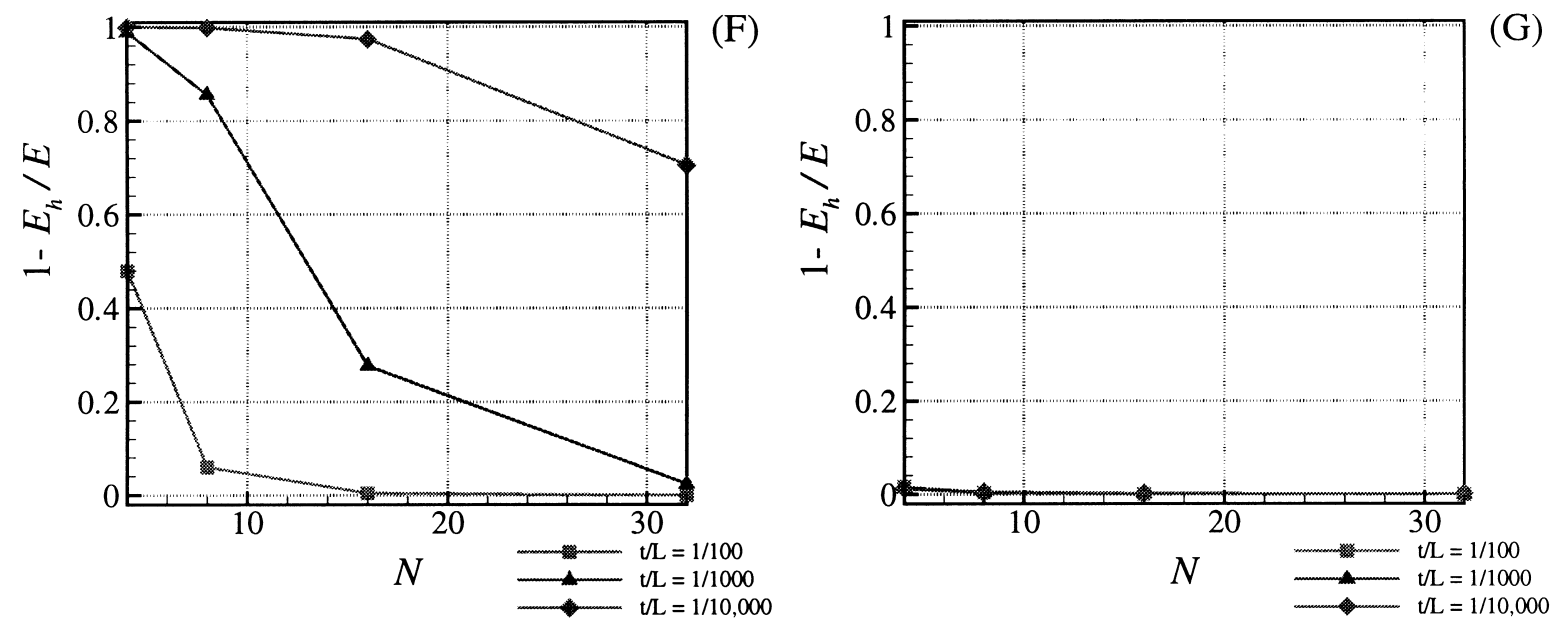

Fig. 17 (continued)

meshes for all the thickness values. More accurate results could not be obtained for the case $t / L=$ $1 / 10,000$ because of round-off errors as a result of illconditioning. This solution difficulty is more likely to arise in bending-dominated problems. In these analyses, pure bending and membrane modes are associated with large differences in stiffness values. Note that this difficulty is less likely to arise when using displacement-based elements because these elements do not accurately represent the pure bending modes.

As in the clamped cylinder analysis, for the $t / L=$ $1 / 100$ case the convergence curves flatten out at the level of $0.01 \%$ because of the discrepancies between the Naghdi theory solution and our three-dimensional shell model.

Figs. 19 and 20 report the performance of the elements when used with the distorted meshes of Fig. 8. Overall, all the discretizations produce worse results compared to uniform meshes. This effect is especially pronounced for coarse meshes with highest distortions. However, as the meshes are refined, the elements reach the accuracy levels obtained with uniform meshes.

\subsubsection{Partly clamped hyperbolic paraboloid}

The problem was suggested in [1] as a good test for locking behavior. The surface is defined as

$Z=X^{2}-Y^{2} ;(X, Y) \in[(-L / 2 ; L / 2)]^{2}$,

clamped along the side $X=-L / 2$ and loaded by self-weight.

By symmetry, only one half of the surface needs to be considered in the analysis (shaded region
$\mathrm{ABCD}$ in Fig. 21), with clamped boundary conditions along $\mathrm{AD}$ and symmetry conditions along AB.

For the finite element analysis we use sequences of $N \times N / 2$ meshes, where $N$ is the number of subdivisions along the $X$-axis. A typical $16 \times 8$ mesh of four-node elements is shown in Fig. 21. These meshes are aligned with the sides of the discretized domain, and not with the asymptotic directions [1].

The analytical solution for this problem is not available, and we have to study the convergence to reference strain energy values obtained with a very fine mesh of shell finite elements. Table 2 reports such results for the strain energy and vertical displacement at point $X=L / 2, Y=0$, calculated using a $48 \times 24$ mesh of MITC16 elements. Note that since the self-weight loading is proportional to thickness, the strain energy scales as $1 / t$, while displacements scale as $1 / t^{2}$.

Figs. 22 and 23 report the convergence in strain energy to the values of Table 2. As in the previous case, the nine-node displacement-based element displays severe locking - for $t / L=1 / 10,000$ the error in strain energy stays above $90 \%$ even for the finest mesh of $32 \times 16$ elements, and its convergence constant $c$ noticeably shifts with variations in thickness.

A milder form of locking can be also observed for the QUAD16 element - its convergence constant increases by an order of magnitude for every decrease in $t$.

The MITC4 element produces good results on the absolute scale, however, the graphs in the logarithmic scale (Fig. 23(A)) show slow convergence. These diffi- 

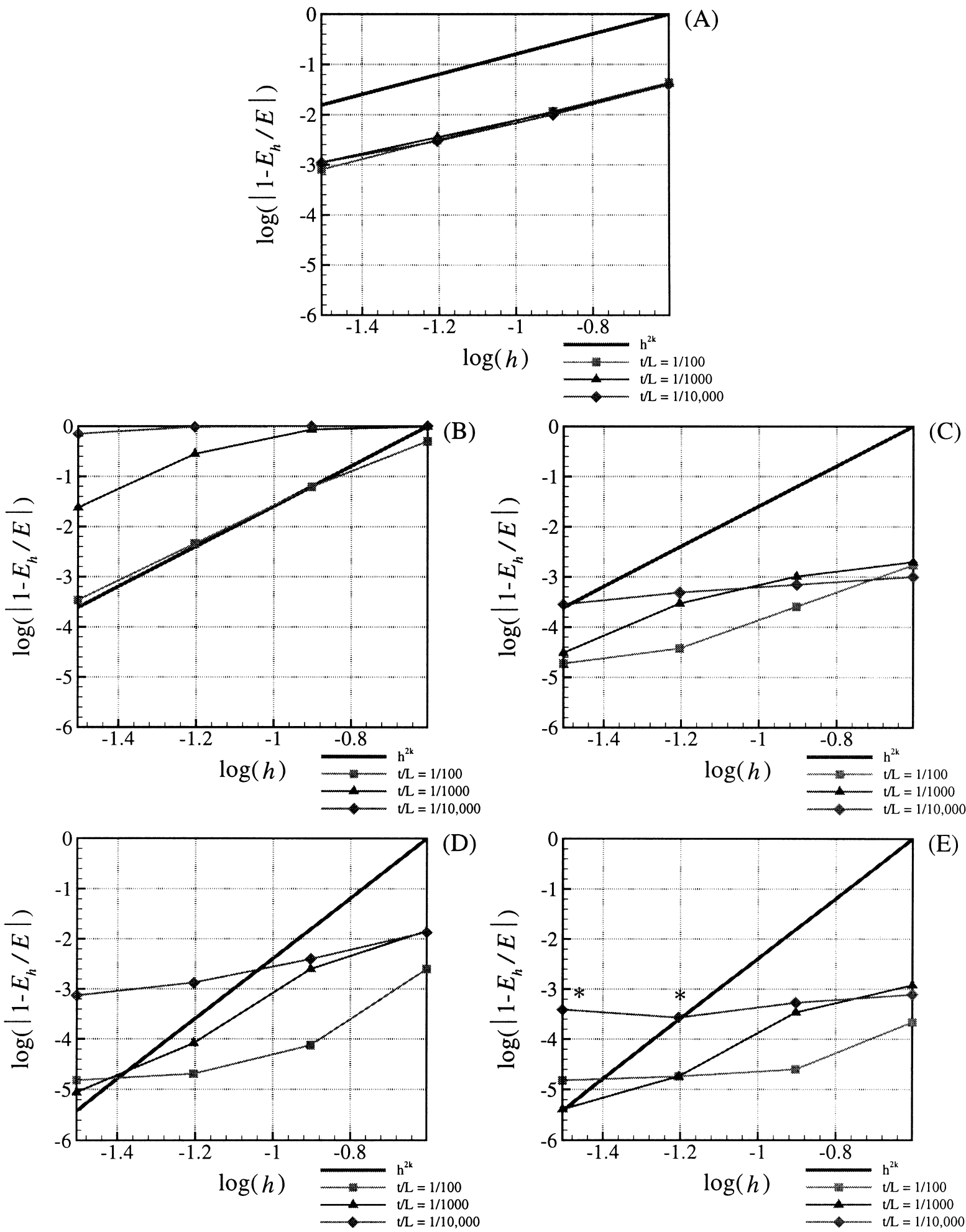

Fig. 18. Convergence in strain energy for the free cylinder problem. Uniform meshes. (A) MITC4 element; (B) QUAD9; (C) MITC9; (D) QUAD16; (E) MITC16; (F) TRI6; (G) MITC6. 

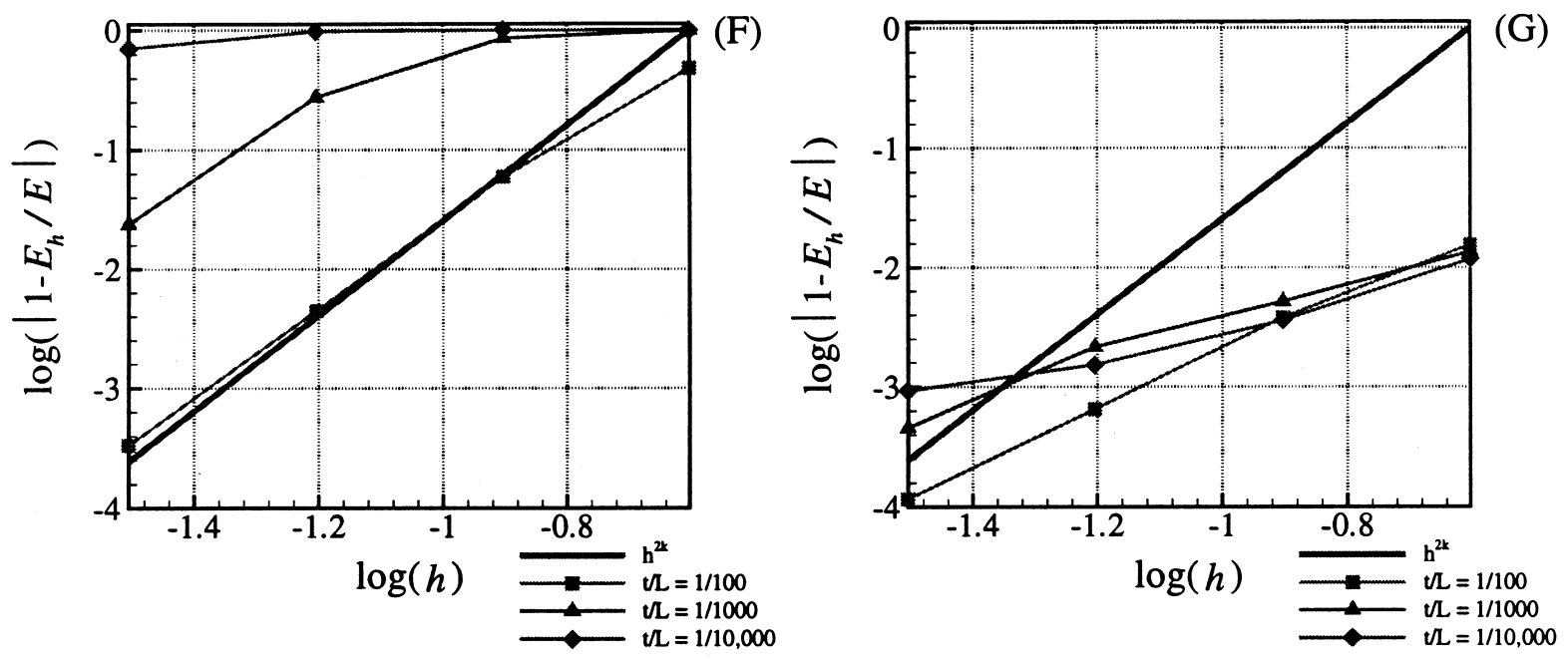

Fig. 18 (continued)

culties can be explained by poor geometric approximation properties achieved with low-order elements for complex surface geometries.

The higher-order MITC elements show good convergence with little dependence on changes in the thickness of the shell structure.

\section{Concluding remarks}

In this paper we have presented a study of the convergence behavior of the MITC shell elements. The problems used in the convergence study were discussed in detail in Ref. [1].

The evaluation of the MITC shell elements shows that the elements are effective in membrane- and bending-dominated shell problems and can thus be employed in general shell analysis situations.

For the membrane-dominated problems, the MITC elements produce results no worse and often significantly better than the displacement-based elements, and show robust convergence properties with low dependence on the thickness parameter $t$.

For the bending-dominated problems, displacementbased elements, of course, exhibit severe locking, while the MITC elements show good performance and robustness.

The optimal convergence rate has not necessarily been observed, but this is probably largely due to boundary layers (as shown in the case of the membrane-dominated cylinder problem).

Regarding the test problems, we have made the following general observations:
- The clamped cylinder problem has proved to be an effective test for membrane-dominated behavior. However, the presence of boundary layers at the fixed edges might induce (localized) instabilities in numerical solutions and makes interpretation of numerical results somewhat difficult.

To avoid this complication, graded meshes refined in the boundary layer regions can be employed.

- The clamped hemispherical cap problem can be used to test the performance of quadrilateral elements but the elements will be quite distorted if the complete shell is discretized. Of course, only a section of a few degrees could be used. The advantage of the problem is that a doubly-curved shell is considered and in practice, of course, distorted elements are commonly used.

- The free cylinder problem should be considered as the first and basic test for locking in bending-dominated situations. Indeed, the space of pure bending displacements $\mathscr{U}_{0}$ has a simple functional (polynomial) form, and thus only elements with relatively poor approximation properties would exhibit locking.

- From our experience, the partly clamped hyperbolic paraboloid problem is an excellent test for locking in bending-dominated situations. The geometry of the shell is more general than a surface of zero Gaussian curvature and the problem is more realistic than an elliptic surface with free boundary. In addition, the symmetry in the problem allows to use relatively fine meshes, and thus, obtain sound convergence curves. 

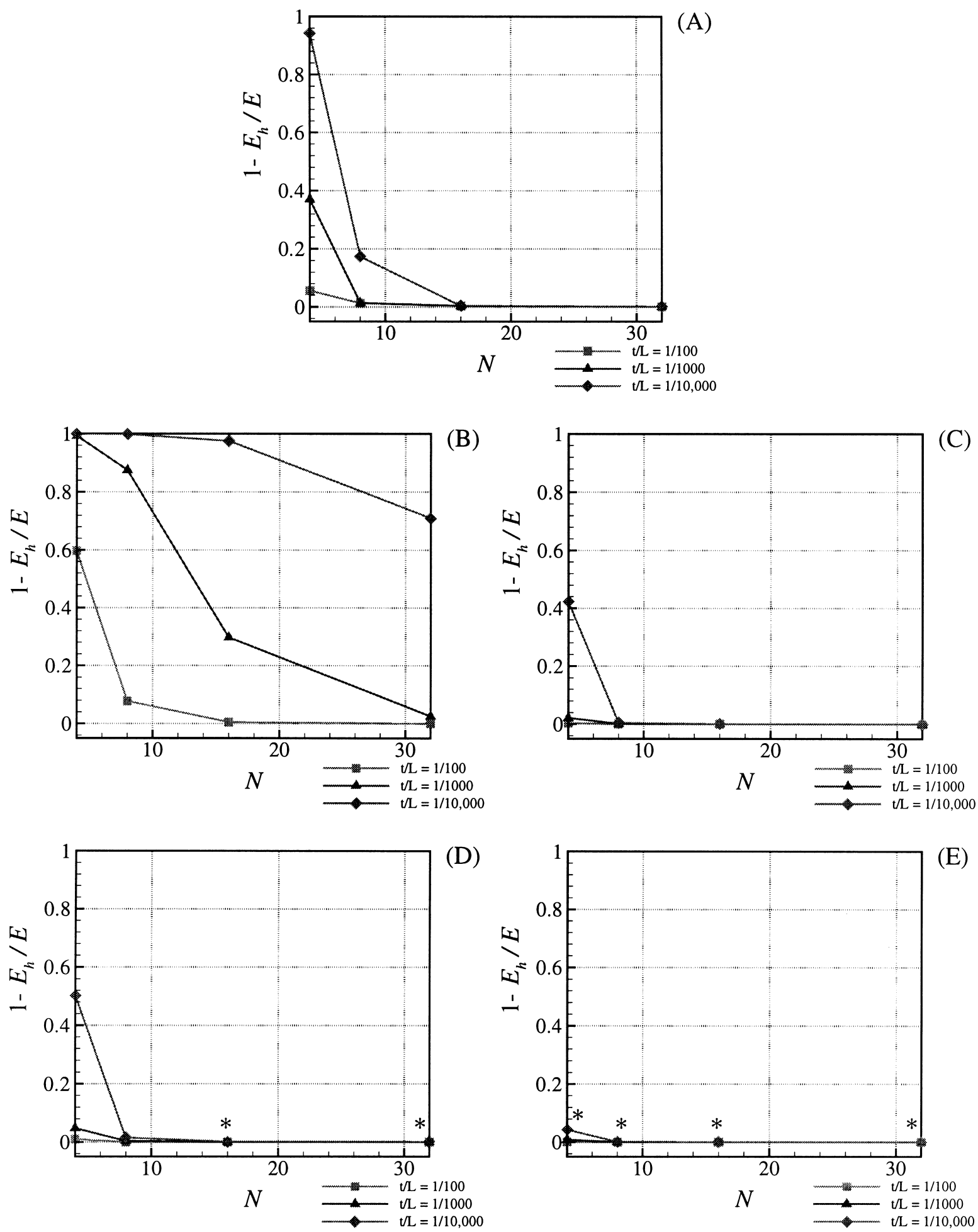

Fig. 19. Convergence in strain energy for the free cylinder problem. Distorted meshes. (A) MITC4 element; (B) QUAD9; (C) MITC9; (D) QUAD16; (E) MITC16. 

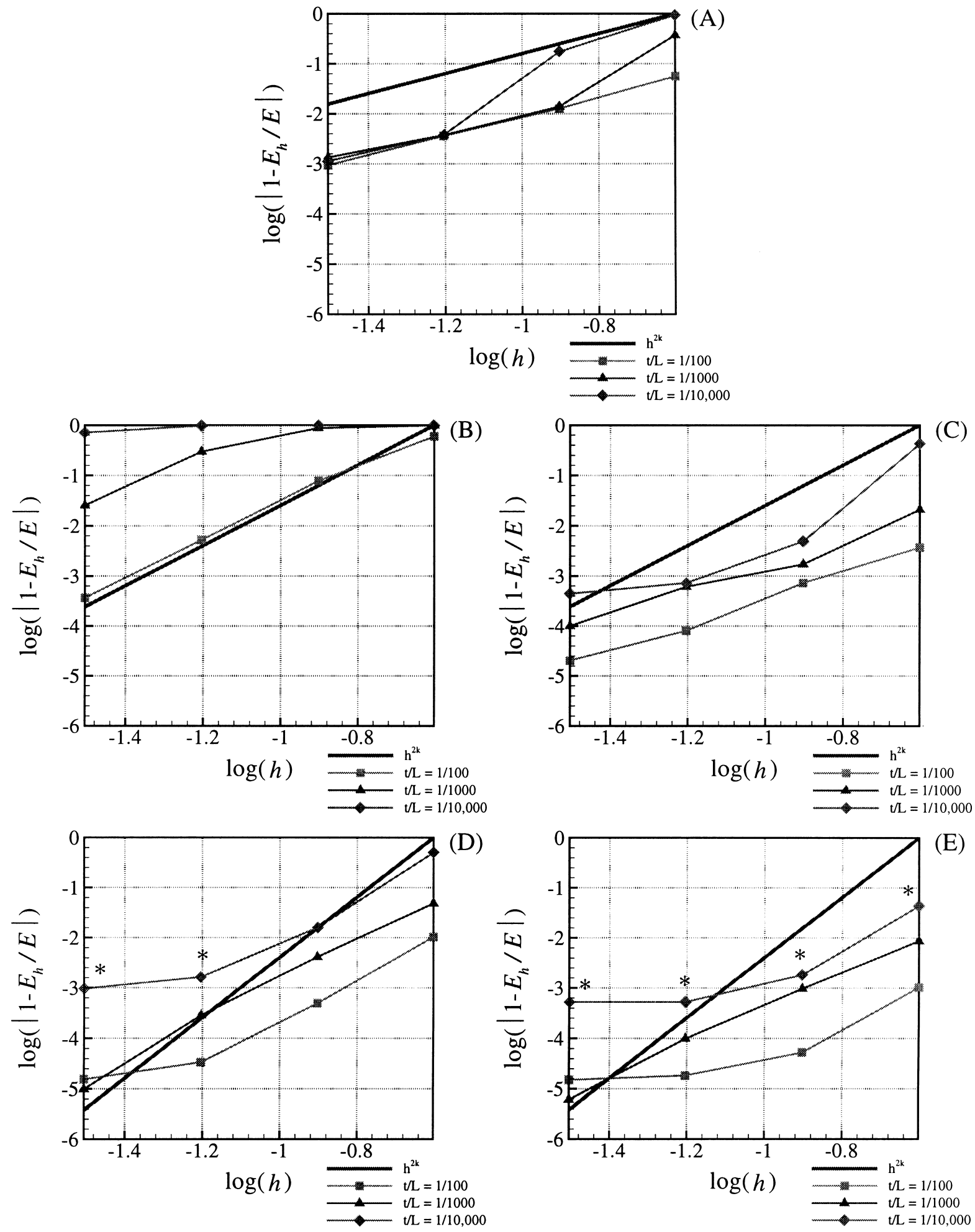

Fig. 20. Convergence in strain energy for the free cylinder problem. Distorted meshes. (A) MITC4 element; (B) QUAD9; (C) MITC9; (D) QUAD16; (E) MITC16. 


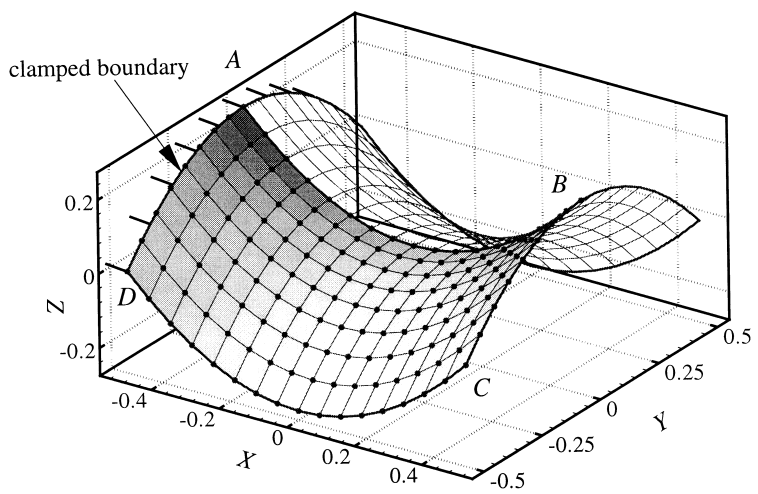

Fig. 21. Partly clamped hyperbolic paraboloid. A $16 \times 8$ mesh of four-node elements is shown.

Based on the results given in Ref. [1] and this paper, we conclude that the test problems used herein are very valuable to evaluate the performance of general shell elements.

\section{Appendix A. Summary of MITC shell element interpolations}

Let $\hat{K}$ denote the area of a reference element. Using the notation already defined, the spaces used for the elements are then as follows.

\section{A.1. The MITC4 element}

The following functional spaces are used for the four-node element:

$$
\begin{aligned}
& \mathscr{V}^{h}=\left\{\boldsymbol{v} \in\left[H^{1}(\Omega)\right]^{3},\left.\boldsymbol{v}\right|_{\hat{K}} \in\left[Q_{1}(\hat{K})\right]^{3}\right\}, \\
& \mathscr{B}^{h}=\left\{\boldsymbol{\eta} \in\left[H^{1}(\Omega)\right]^{2},\left.\boldsymbol{\eta}\right|_{\hat{K}} \in\left[Q_{1}(\hat{K})\right]^{2}\right\}, \\
& \mathscr{E}_{13}^{h h}=\left\{\psi \in L^{2}(\Omega),\left.\psi\right|_{\hat{K}} \in \operatorname{span}\left\{1, \hat{\xi}_{2}\right\}\right\}, \\
& \mathscr{E}_{23}^{h}=\left\{\psi \in L^{2}(\Omega),\left.\psi\right|_{\hat{K}} \in \operatorname{span}\left\{1, \hat{\xi}_{1}\right\}\right\} .
\end{aligned}
$$

The tying procedure for the transverse shear strains $\varepsilon_{13}$ and $\varepsilon_{23}$ is shown in Fig. A1(A).

\section{A.2. The MITC9 element}

The following functional spaces are used to con- struct the nine-node element:

$$
\begin{aligned}
\mathscr{V}^{h} & =\left\{\boldsymbol{v} \in\left[H^{1}(\Omega)\right]^{3},\left.\boldsymbol{v}\right|_{\hat{K}} \in\left[Q_{2}(\hat{K})\right]^{3}\right\}, \\
\mathscr{B}^{h} & =\left\{\boldsymbol{\eta} \in\left[H^{1}(\Omega)\right]^{2},\left.\boldsymbol{\eta}\right|_{\hat{K}} \in\left[Q_{2}(\hat{K})\right]^{2}\right\}, \\
\mathscr{E}_{11}^{h h} & =\mathscr{E}_{13}^{h h}=\left\{\psi \in L^{2}(\Omega),\left.\psi\right|_{\hat{K}}\right. \\
& \left.\in \operatorname{span}\left\{1, \hat{\xi}_{1}, \hat{\xi}_{2}, \hat{\xi}_{1} \hat{\xi}_{2}, \hat{\xi}_{2}^{2}, \hat{\xi}_{1} \hat{\xi}_{2}^{2}\right\}\right\},
\end{aligned}
$$

$$
\begin{aligned}
\mathscr{E}_{22}^{h} & =\mathscr{E}_{23}^{h h}=\left\{\psi \in L^{2}(\Omega),\left.\psi\right|_{\hat{K}}\right. \\
& \left.\in \operatorname{span}\left\{1, \hat{\xi}_{1}, \hat{\xi}_{2}, \hat{\xi}_{1} \hat{\xi}_{2}, \hat{\xi}_{1}^{2}, \hat{\xi}_{1}^{2} \hat{\xi}_{2}\right\}\right\} .
\end{aligned}
$$

$\mathscr{E}_{12}^{h}=\left\{\psi \in L^{2}(\Omega),\left.\psi\right|_{\hat{K}} \in Q_{1}(\hat{K})\right\}$.

The tying procedure for the element is given in Fig. A1(B).

\section{A.3. The MITC16 element}

The 16-node cubic element is constructed using:

$$
\begin{aligned}
\mathscr{V}^{h} & =\left\{\boldsymbol{v} \in\left[H^{1}(\Omega)\right]^{3},\left.\boldsymbol{v}\right|_{\hat{K}} \in\left[Q_{3}(\hat{K})\right]^{3}\right\}, \\
\mathscr{B}^{h} & =\left\{\boldsymbol{\eta} \in\left[H^{1}(\Omega)\right]^{2},\left.\boldsymbol{\eta}\right|_{\hat{K}} \in\left[Q_{3}(\hat{K})\right]^{2}\right\}, \\
\mathscr{E}_{11}^{h h} & =\mathscr{E}_{13}^{h h}=\left\{\psi \in L^{2}(\Omega),\left.\psi\right|_{\hat{K}}\right. \\
& \in \operatorname{span}\left\{1, \hat{\xi}_{1}, \hat{\xi}_{2}, \hat{\xi}_{1}^{2}, \hat{\xi}_{1} \hat{\xi}_{2}, \hat{\xi}_{2}^{2}, \hat{\xi}_{1}^{2} \hat{\xi}_{2}, \hat{\xi}_{1} \hat{\xi}_{2}^{2}, \hat{\xi}_{2}^{3}, \hat{\xi}_{1}^{2} \hat{\xi}_{2}^{2}\right. \\
, & \left.\left.\hat{\xi}_{1} \hat{\xi}_{2}^{3}, \hat{\xi}_{1}^{2} \hat{\xi}_{2}^{3}\right\}\right\}, \\
\mathscr{E}_{22}^{h h} & =\mathscr{E}_{23}^{h h}=\left\{\psi \in L^{2}(\Omega),\left.\psi\right|_{\hat{K}}\right. \\
& \in \operatorname{span}\left\{1, \hat{\xi}_{1}, \hat{\xi}_{2}, \hat{\xi}_{1}^{2}, \hat{\xi}_{1} \hat{\xi}_{2}, \hat{\xi}_{2}^{2}, \hat{\xi}_{1}^{3}, \hat{\xi}_{1}^{2} \hat{\xi}_{2}, \hat{\xi}_{1} \hat{\xi}_{2}^{2}\right. \\
, & \left.\left.\hat{\xi}_{1}^{3} \hat{\xi}_{2}, \hat{\xi}_{1}^{2} \hat{\xi}_{2}^{2}, \hat{\xi}_{1}^{3} \hat{\xi}_{2}^{2}\right\}\right\} .
\end{aligned}
$$

$\mathscr{E}_{12}^{\mathscr{h}_{12}}=\left\{\psi \in L^{2}(\Omega),\left.\psi\right|_{\hat{K}} \in Q_{2}(\hat{K})\right\}$. 

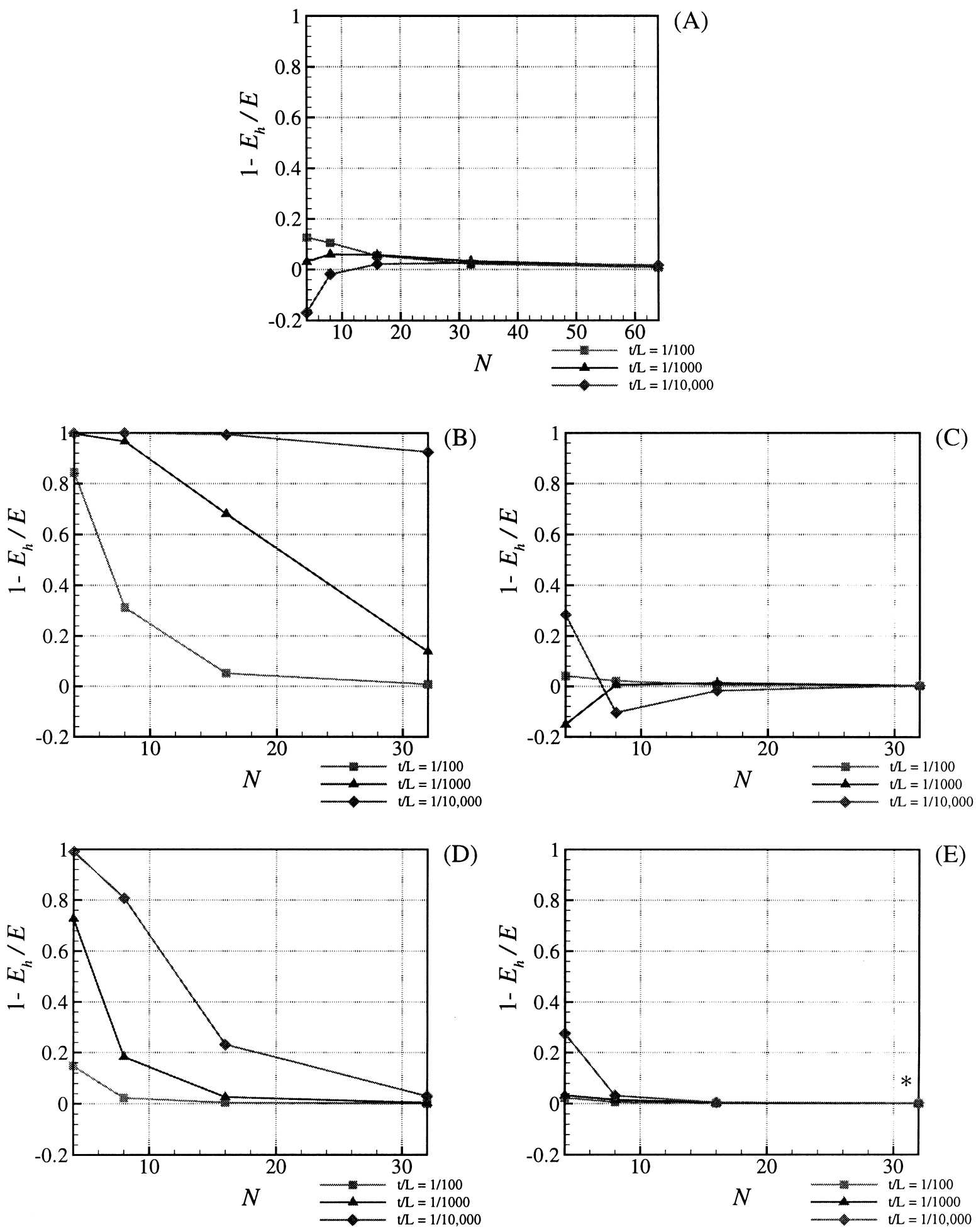

Fig. 22. Convergence in strain energy for the hyperbolic paraboloid problem. (A) MITC4 element; (B) QUAD9; (C) MITC9; (D) QUAD16; (E) MITC16; (F) TRI6; (G) MITC6. 

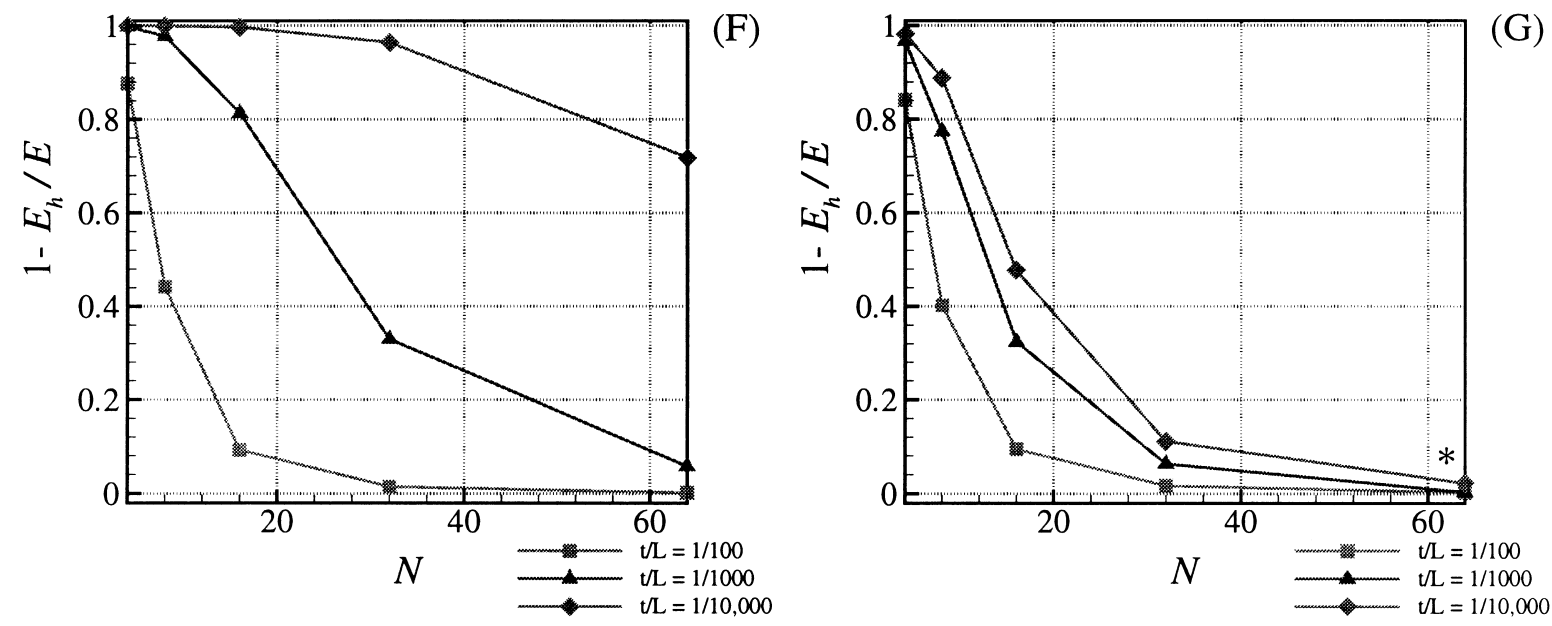

Fig. 22 (continued)

The tying procedure for the strain tensor components is presented in Fig. A1(C).

\section{A.4. The MITC6 element}

The six-node triangular element is constructed using

$\mathscr{V}^{h}=\left\{v \in\left[H^{1}(\Omega)\right]^{3},\left.v\right|_{\hat{K}} \in\left[P_{2}(\hat{K})\right]^{3}\right\}$,

$\mathscr{B}^{h}=\left\{\boldsymbol{\eta} \in\left[H^{1}(\Omega)\right]^{2},\left.\boldsymbol{\eta}\right|_{\hat{K}} \in\left[P_{2}(\hat{K})\right]^{2}\right\}$

$\mathscr{E}_{11}^{\mathscr{C}_{h}}=\mathscr{E}_{12}^{\mathscr{E}_{h}}=\mathscr{E}_{22}^{\mathscr{h}_{h}}=\left\{\psi \in L^{2}(\Omega),\left.\psi\right|_{\hat{K}} \in P_{1}(\Omega)\right\}$,

$\mathscr{E}_{13}^{\mathscr{E}^{h}}=\left\{\psi \in L^{2}(\Omega),\left.\psi\right|_{\hat{K}} \in \operatorname{span}\left\{1, \hat{\xi}_{1}, \hat{\xi}_{1} \hat{\xi}_{2}, \hat{\xi}_{2}, \hat{\xi}_{2}^{2}\right\}\right\}$

$\mathscr{E}_{23}^{h}=\left\{\psi \in L^{2}(\Omega),\left.\psi\right|_{\hat{K}} \in \operatorname{span}\left\{1, \hat{\xi}_{1}, \hat{\xi}_{1} \hat{\xi}_{2}, \hat{\xi}_{2}, \hat{\xi}_{1}^{2}\right\}\right\}$.

The choice of interpolation spaces for the transverse shear components proved to be optimal for the mixedinterpolated seven-node plate bending element MITC7 [2]. The tying procedure for the strain tensor components is depicted in Fig. A2.

\section{Appendix B. Mixed formulation for the MITC general shell elements}

In this Appendix we demonstrate how the abstract variational problem of type (15) can be derived from the mixed interpolation employed in construction of the general MITC shell elements using the HellingerReissner variational principle.

Using the engineering notation [2], we rewrite the strain tensor in vector form:

$\boldsymbol{\varepsilon}=\left[\varepsilon_{11}, \varepsilon_{22}, \varepsilon_{33}, 2 \varepsilon_{12}, 2 \varepsilon_{23}, 2 \varepsilon_{13}\right]^{\mathrm{T}}$,

and assume

$\varepsilon=\varepsilon^{\mathrm{DB}}+\varepsilon^{\mathrm{AS}}$

The vector $\varepsilon^{\mathrm{DB}}$ contains the components of the strain vector $\boldsymbol{\varepsilon}$ that are directly calculated from displacements, while vector $\boldsymbol{\varepsilon}^{\mathrm{AS}}$ contains the components which are obtained using some assumed strain interpolation. Let the complete vector of strains obtained from the displacements be $\overline{\boldsymbol{\varepsilon}}$, that is:

$\overline{\boldsymbol{\varepsilon}}=\left[\varepsilon_{11}(\boldsymbol{U}), \varepsilon_{22}(\boldsymbol{U}), \varepsilon_{33}(\boldsymbol{U}), 2 \varepsilon_{12}(\boldsymbol{U}), 2 \varepsilon_{23}(\boldsymbol{U}), 2 \varepsilon_{13}(\boldsymbol{U})\right]^{\mathrm{T}}$

then

$\overline{\boldsymbol{\varepsilon}}=\boldsymbol{\varepsilon}^{\mathrm{DB}}+\boldsymbol{\varepsilon}^{\mathrm{TI}}$,

where $\boldsymbol{\varepsilon}^{\mathrm{TI}}$ contains the displacement-based strain components to be tied to the assumed strain components $\varepsilon^{\mathrm{AS}}$.

The Hellinger-Reissner variational indicator can be then written as [2] (neglecting the loading terms): 

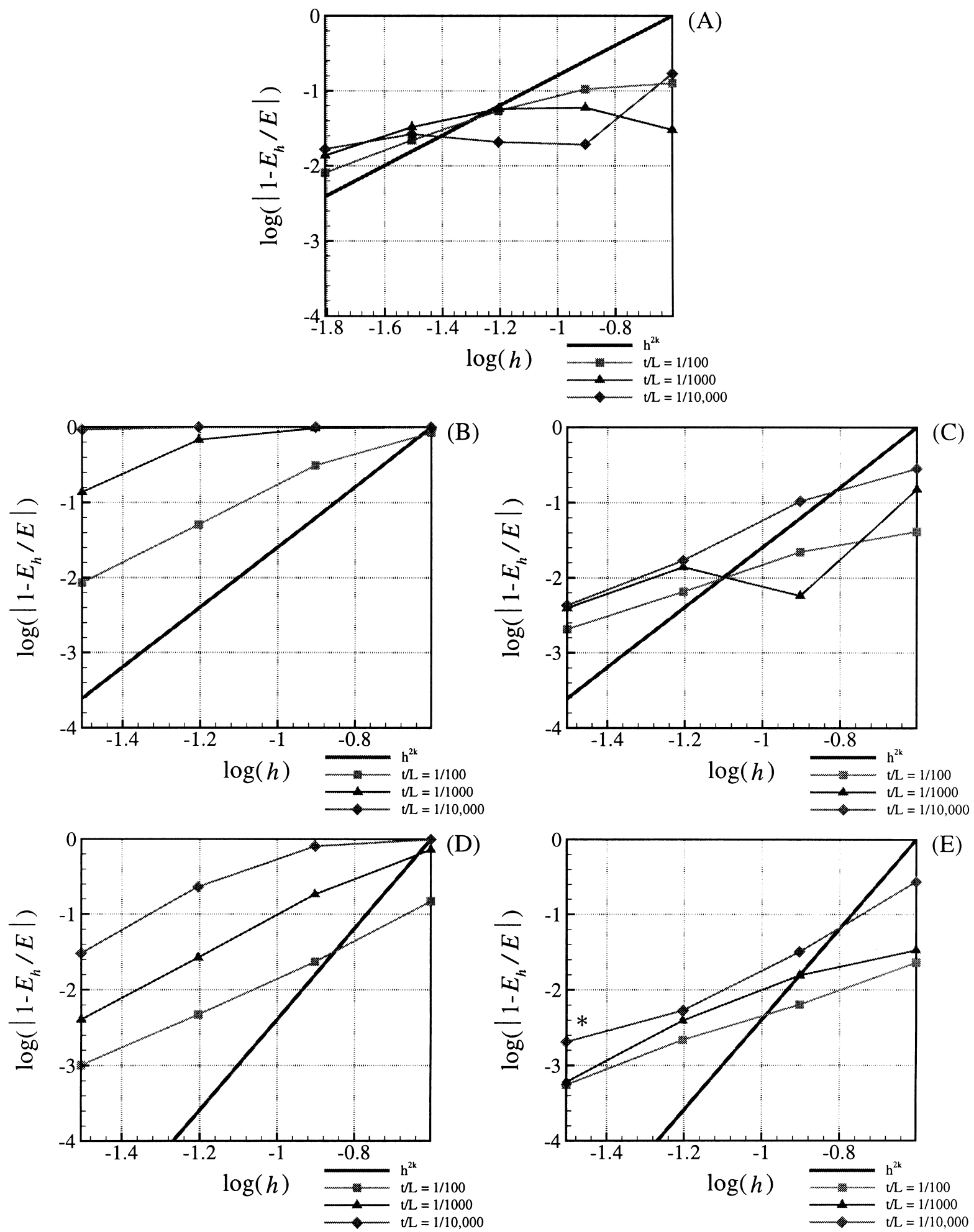

Fig. 23. Convergence in strain energy for the hyperbolic paraboloid problem. (A) MITC4 element; (B) QUAD9; (C) MITC9; (D) QUAD16; (E) MITC16; (F) TRI6; (G) MITC6. 

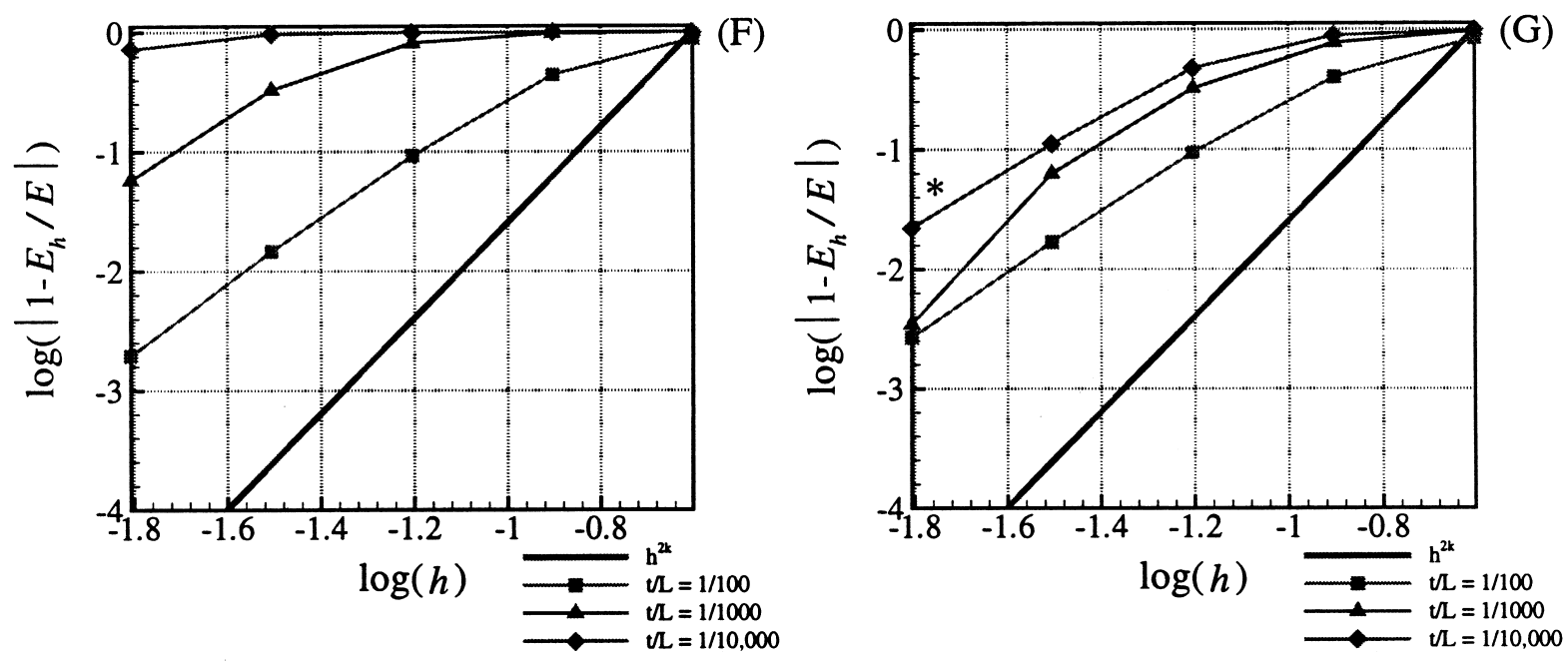

Fig. 23 (continued)

(A)

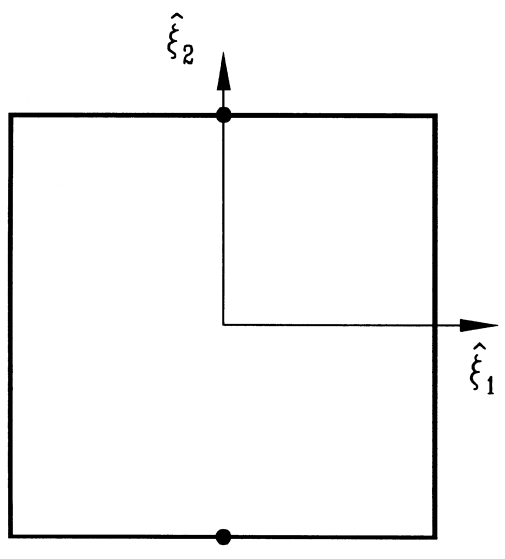

(C)

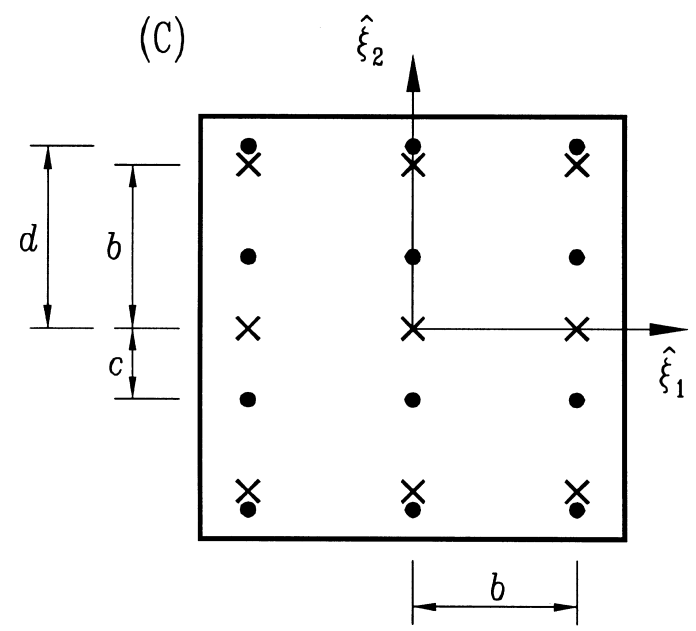

(B)

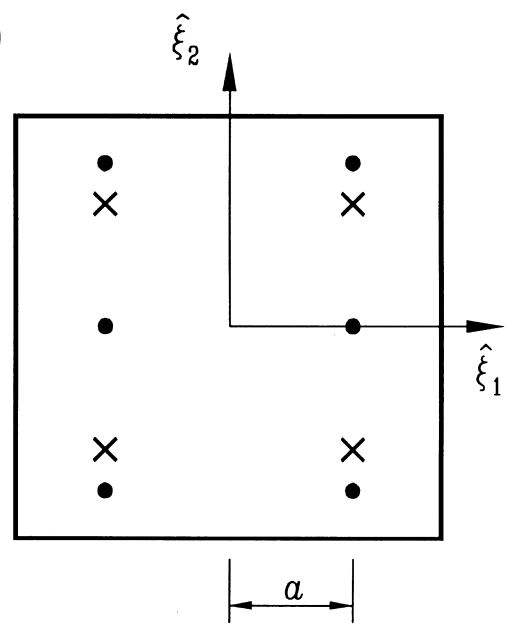

Fig. A1. Tying points for the MITC shell elements. (A) MITC4 element; (B) MITC9 element; (C) MITC16 element. 

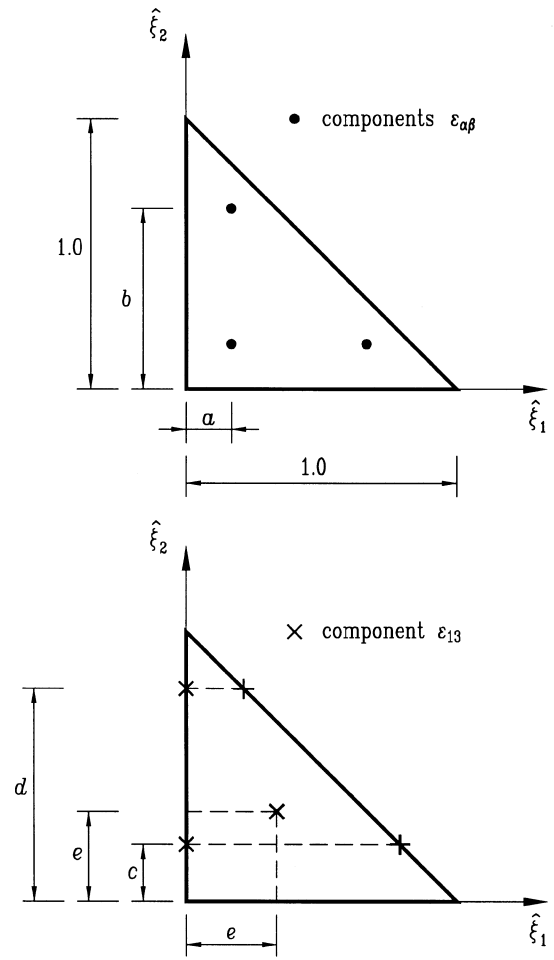

Fig. A2. Tying points for the six-node mixed-interpolated shell element MITC6.

$$
\begin{aligned}
\Pi_{\mathrm{HR}}(\boldsymbol{U}, \boldsymbol{\varepsilon})= & -\frac{1}{2} \int_{V} \boldsymbol{\varepsilon}^{\mathrm{T}} \boldsymbol{C} \boldsymbol{\varepsilon} \mathrm{d} V+\int_{V} \boldsymbol{\varepsilon}^{\mathrm{T}} \boldsymbol{C} \overline{\boldsymbol{\varepsilon}} \mathrm{d} V \\
= & \frac{1}{2} \int_{V}\left(\boldsymbol{\varepsilon}^{\mathrm{DB}}\right)^{\mathrm{T}} \boldsymbol{C} \boldsymbol{\varepsilon}^{\mathrm{DB}} \mathrm{d} V \\
& -\frac{1}{2} \int_{V}\left(\boldsymbol{\varepsilon}^{\mathrm{AS}}\right)^{\mathrm{T}} \boldsymbol{C} \boldsymbol{\varepsilon}^{\mathrm{AS}} \mathrm{d} V \\
& +\int_{V}\left(\boldsymbol{\varepsilon}^{\mathrm{DB}}\right)^{\mathrm{T}} \boldsymbol{C} \boldsymbol{\varepsilon}^{\mathrm{TI}} \mathrm{d} V \\
& +\int_{V}\left(\boldsymbol{\varepsilon}^{\mathrm{AS}}\right)^{\mathrm{T}} \boldsymbol{C} \boldsymbol{\varepsilon}^{\mathrm{TI}} \mathrm{d} V
\end{aligned}
$$

where $\boldsymbol{C}$ is the matrix of constitutive relations.

Invoking the stationarity conditions for $\Pi_{\mathrm{HR}}$ with respect to $\boldsymbol{U}$ and $\boldsymbol{\varepsilon}^{\mathrm{AS}}$,

$\delta \Pi_{\mathrm{HR}}\left(\boldsymbol{U}, \boldsymbol{\varepsilon}^{\mathrm{AS}}\right)=0$

we obtain:

$$
\begin{aligned}
& \int_{V}\left(\delta \boldsymbol{\varepsilon}^{\mathrm{DB}}\right)^{\mathrm{T}} \boldsymbol{C} \overline{\boldsymbol{\varepsilon}} \mathrm{d} V+\int_{V}\left(\delta \boldsymbol{\varepsilon}^{\mathrm{TI}}\right)^{\mathrm{T}} \boldsymbol{C} \boldsymbol{\varepsilon} \mathrm{d} V=0, \\
& \int_{V}\left(\delta \boldsymbol{\varepsilon}^{\mathrm{AS}}\right)^{\mathrm{T}} \boldsymbol{C}\left(\boldsymbol{\varepsilon}^{\mathrm{TI}}-\boldsymbol{\varepsilon}^{\mathrm{AS}}\right) \mathrm{d} V=0 .
\end{aligned}
$$

Note that when all the components of the strain tensor are tied, i.e., $\boldsymbol{\varepsilon}^{\mathrm{DB}}=\delta \boldsymbol{\varepsilon}^{\mathrm{DB}}=0$, the formulation becomes:

$$
\begin{aligned}
& \int_{V}\left(\delta \boldsymbol{\varepsilon}^{\mathrm{TI}}\right)^{\mathrm{T}} \boldsymbol{C} \boldsymbol{\varepsilon}^{\mathrm{AS}} \mathrm{d} V=0, \\
& \int_{V}\left(\delta \boldsymbol{\varepsilon}^{\mathrm{AS}}\right)^{\mathrm{T}} \boldsymbol{C}\left(\boldsymbol{\varepsilon}^{\mathrm{TI}}-\boldsymbol{\varepsilon}^{\mathrm{AS}}\right) \mathrm{d} V=0 .
\end{aligned}
$$

This mixed formulation pertaining to the underlying mathematical model (discussed in Ref. [6]) is the formulation in Eq. (15) for the Naghdi shell model.

The second line of Eq. (B7) defines the "tying" procedure by which $\varepsilon^{\mathrm{AS}}$ is obtained from $\boldsymbol{\varepsilon}^{\mathrm{TI}}$. For the MITC elements we directly use $\varepsilon^{\mathrm{AS}}=\mathscr{J}\left(\boldsymbol{\varepsilon}^{\mathrm{TI}}\right)$, where $\mathscr{J}\left(\boldsymbol{\varepsilon}^{\mathrm{TI}}\right)$ denotes the assumed interpolation of $\boldsymbol{\varepsilon}^{\mathrm{TI}}$ in the strain space. Hence, the integral form of Eq. (B8) is equivalent to the MITC formulation if

$\int_{V}\left(\delta \boldsymbol{\varepsilon}^{\mathrm{AS}}\right)^{\mathrm{T}} \boldsymbol{C} \boldsymbol{\varepsilon}^{\mathrm{TI}} \mathrm{d} V=\int_{V}\left(\delta \boldsymbol{\varepsilon}^{\mathrm{AS}}\right)^{\mathrm{T}} \boldsymbol{C} \mathscr{J}\left(\boldsymbol{\varepsilon}^{\mathrm{TI}}\right) \mathrm{d} V$.

This relation holds true if the integral on the left-hand side can be exactly evaluated by using the values of $\delta \varepsilon^{\mathrm{AS}}$ and $\boldsymbol{\varepsilon}^{\mathrm{TI}}$ at the tying points only. Considering plane elements, this clearly holds for rectangular MITC9 and MITC16 elements for which the tying points correspond to Gauss integration points that can be used to integrate the quantity $\left(\delta \boldsymbol{\varepsilon}^{\mathrm{AS}}\right)^{\mathrm{T}} \boldsymbol{C} \boldsymbol{\varepsilon}^{\mathrm{TI}}$ exactly. Eq. (B9) can be shown to hold for the MITC4 and MITC6 elements as well by computing both sides analytically (using symbolic calculus software, for example).

\section{References}

[1] Chapelle D, Bathe KJ. Fundamental considerations for the finite element analysis of shell structures. Computers and Structures 1998;66:19-36.

[2] Bathe KJ. Finite element procedures. Englewood Cliffs, NJ: Prentice-Hall, 1996.

[3] Dvorkin EN, Bathe KJ. A continuum mechanics based four-node shell element for general nonlinear analysis. Eng Comput 1984;1:77-88.

[4] Bucalem ML, Bathe KJ. Higher-order MITC general shell elements. Int J Num Meth Eng 1993;36:3729-54.

[5] Naghdi PM. Foundations of elastic shell theory. In: Progress in solid mechanics, vol. 4. Amsterdam: NorthHolland, 1963. p. 1-90.

[6] Chapelle D, Bathe KJ. On general shell finite elements and mathematical shell models. In: Topping BHV, editor. Advances in finite element procedures and techniques. Edinburgh, Scotland: Civil-Comp Press, 1998. p. 25-30.

[7] Bathe KJ, Iosilevich A, Chapelle D. An inf-sup test for shell finite elements. Computers and Structures (in press).

[8] Pitkäranta J, Leino Y, Ovaskainen O, Piila J. Shell deformation states and the finite element method: a benchmark study of cylindrical shells. Comp Meth Appl Mech Eng 1995; 128:81-121. 